
Cahiers
de a Recherche
Fur les Droits
Fondamentaux
Cahiers de la recherche sur les droits fondamentaux

$13 \mid 2015$

Le droit d'asile

\title{
Chronique de jurisprudence constitutionnelle française 2014
}

Journal of French Constitutional Case Law 2014

Quentin Butavand, Eugénie Duval, Alexandra Korsakoff, David Poinsignon et David Vicomte

\section{(2) OpenEdition}

Édition électronique

URL : http://journals.openedition.org/crdf/1278

DOI : $10.4000 /$ crdf. 1278

ISSN : 2264-1246

Éditeur

Presses universitaires de Caen

Édition imprimée

Date de publication : 1 novembre 2015

Pagination : 147-162

ISBN : 978-2-84133-742-2

ISSN : $1634-8842$

Référence électronique

Quentin Butavand, Eugénie Duval, Alexandra Korsakoff, David Poinsignon et David Vicomte, «Chronique de jurisprudence constitutionnelle française 2014 ». Cahiers de la recherche sur les droits fondamentaux [En ligne], 13 | 2015, mis en ligne le 01 novembre 2016, consulté le 11 février 2020. URL : http://journals.openedition.org/crdf/1278; DOI : 10.4000/crdf.1278 


\title{
Chronique de jurisprudence constitutionnelle française 2014
}

\author{
Quentin BUTAVAND \\ Doctorant en droit public à l'université Paris Ouest Nanterre La Défense \\ Centre de recherches et d'études sur les droits fondamentaux (CREDOF, EA 3933) \\ Chercheur associé au Centre de recherche sur les droits fondamentaux et les évolutions du droit (CRDFED, EA 2132)
}

Eugénie DUVAL

Doctorante en droit public à l'université de Caen Normandie

Centre de recherche sur les droits fondamentaux et les évolutions du droit (CRDFED, EA 2132)

\section{Alexandra KORSAKOFF}

Doctorante en droit public à l'université de Caen Normandie

Centre de recherche sur les droits fondamentaux et les évolutions du droit (CRDFED, EA 2132)

\section{David POINSIGNON}

Doctorant en droit public à l'université de Caen Normandie

Centre de recherche sur les droits fondamentaux et les évolutions du droit (CRDFED, EA 2132)

\section{David VICOMTE}

Doctorant en droit public à I'université de Caen Normandie

Centre de recherche sur les droits fondamentaux et les évolutions du droit (CRDFED, EA 2132)

I. Le contrôle formel

A. Les modalités du contrôle de constitutionnalité

1. La contestation de la constitutionnalité des lois encadrée

2. Des conditions de fond à respecter

B. Le contrôle de la qualité de la loi

1. Un législateur tenu d'exercer sa propre compétence

2. Le contrôle de la procédure législative

II. Le contrôle substantiel

A. Le droit de propriété

1. La privation du droit de propriété

2. La limitation du droit de propriété

B. La liberté d'entreprendre

1. Une liberté peu autonome

2. Une liberté limitée 
C. L'article 16 de la Déclaration des droits de l'homme et du citoyen

1. L'indépendance et l'impartialité des juridictions

2. Le droit à un recours juridictionnel effectif

3. Le respect des droits de la défense

D. Le principe d'égalité

1. Un traitement traditionnel et uniforme du principe d'égalité:

la référence à l'article 6 de la Déclaration des droits de l'homme et du citoyen de 1789

2. Une innovation dans le cadre de la QPC: censure de l'infériorité des droits reconnus aux femmes

En 2014, le Conseil constitutionnel a quelque peu innové et surtout approfondi sa jurisprudence. Son rôle de gardien de l'État de droit est reconnu par le législateur lui-même. Dans la décision no 2014-693 DC ${ }^{1}$, pour la première fois, les auteurs de la saisine n'étaient pas les détracteurs du texte déféré au Conseil mais la majorité parlementaire qui avait soutenu ce texte. La mise en œuvre du droit de saisine, reconnu aux minorités parlementaires depuis $1974^{2}$, était jusqu'alors essentiellement envisagée comme un moyen d'entrave à la volonté politique de la majorité. Tel n'est pourtant pas le cas dans cette décision: les députés auteurs de la saisine envisagent le rôle du Conseil dans son sens premier, celui d'assurer la suprématie de la Constitution. Par ailleurs, le rôle et les fonctions des membres du Parlement ont été également précisés par une nouvelle formulation issue de la décision $\mathrm{n}^{\circ}$ 2014-407 QPC ${ }^{3}$. D'autres innovations peuvent être notées dans la jurisprudence du Conseil constitutionnel. Pour la première fois, le Conseil a été saisi afin d'apprécier la sincérité d'une loi de financement rectificative de la Sécurité sociale ${ }^{4}$. Par conséquent, un nouveau considérant de principe fut dégagé par le Conseil ${ }^{5}$. L'article 8 de la Déclaration des droits de l'homme et du citoyen (DDHC), duquel découle le principe de légalité des peines, connaît une nouvelle portée. À présent, ce principe «impose au législateur de fixer les sanctions disciplinaires en des termes suffisamment clairs et précis pour exclure l'arbitraire ${ }^{6}$. D'autres innovations et surtout de nombreuses précisions ont permis d'approfondir la jurisprudence des juges de Montpensier, tant sur le plan procédural (I) que matériel (II).

\section{Le contrôle formel}

\section{A. Les modalités du contrôle de constitutionnalité}

Au regard des quelques précisions apportées par le Conseil constitutionnel en 2014, il convient de rappeler brièvement les différentes procédures permettant de contester la constitutionnalité de dispositions législatives (1), ainsi que les conditions à respecter pour contester des dispositions (2).

\section{La contestation de la constitutionnalité des lois encadrée}

La saisine du Conseil constitutionnel est strictement réglementée. Seuls le président de la République, le Premier ministre, le président de l'Assemblée nationale ou du Sénat, ainsi que soixante députés ou soixante sénateurs sont habilités à déférer une loi non promulguée au Conseil 7 .

La situation diffère quelque peu lorsque la loi est déjà en vigueur. Dans cette hypothèse, les juges constitutionnels sont en principe saisis par le Conseil d'État ou la Cour de cassation d'une question prioritaire de constitutionnalité (QPC) soulevée par une partie d'une instance

1. CC, déc. n 2014-693 DC du 25 mars 2014, Loi relative à la géolocalisation.

2. Loi constitutionnelle $\mathrm{n}^{\circ} 74-904$ du 29 octobre 1974 portant révision de l'article 61 de la Constitution, JORF, 30 octobre 1974, p. 11035.

3. CC, déc. $\mathrm{n}^{\mathrm{o}}$ 2014-407 QPC du 18 juillet 2014, MM. Jean-Louis M. et Jacques B., cons. 19: «il ressort [des articles $1^{\text {er }}$ et 3 de la Constitution] que les membres du Parlement ont la qualité de représentants du peuple; qu'en outre, si députés et sénateurs sont élus au suffrage universel, direct pour les premiers, indirect pour les seconds, chacun d'eux représente au Parlement la Nation tout entière et non la population de la circonscription où il a été élu; qu'à ce titre ils sont appelés à voter la loi dans les conditions fixées par la Constitution et les dispositions ayant valeur de loi organique prises pour son application».

4. CC, déc. n 2014-698 DC du 6 août 2014, Loi de financement rectificative de la Sécurité sociale pour 2014.

5. Ibid., cons. 3, le considérant de principe classiquement appliqué aux lois de financement de la Sécurité sociale est pour la première fois appliqué à une loi rectificative.

6. CC, déc. n² 2014-385 QPC du 28 mars 2014, M. Joël M., cons. 6.

7. Est par conséquent irrecevable le recours formulé par un parlementaire, seul, par lequel il demande d’être signataire d'un recours principal formé préalablement par ses homologues, alors même qu'il ne formule pas les mêmes griefs à l'encontre de la loi: CC, déc. n²013-687 DC du 23 janvier 2014, Loi de modernisation de l'action publique territoriale et d'affirmation des métropoles, cons. 2 à 3. 
en cours devant une juridiction ${ }^{8}$. Rappelons qu'une QPC ne peut être formulée devant une cour d'assises ${ }^{9}$, ni dans le cadre d'une simple procédure d'admission à l'aide juridictionnelle ${ }^{10}$. Par exception cependant, le Conseil constitutionnel peut être directement saisi lorsque le Conseil d'État et la Cour de cassation ne statuent pas dans le délai de trois mois imparti ${ }^{11}$. Il s'agit d'un fait assez rare, intervenu quatre fois jusqu'à maintenant ${ }^{12}$, dont une fois cette année. La QPC peut alors être transmise soit par le requérant ${ }^{13}$, soit par la juridiction qui a constaté son dessaisissement ${ }^{14}$.

D’autre part, le mécanisme des interventions permet à des tiers à une procédure déjà engagée devant le Conseil de formuler des observations relatives à la constitutionnalité de la loi soumise à son examen. Formellement, il n'existe que dans le cadre du contentieux du contrôle a posteriori des lois. Formalisée tardivement ${ }^{15}$, cette faculté est aujourd'hui strictement encadrée, car subordonnée à une double condition: lesdits tiers doivent justifier d'un intérêt spécial ${ }^{16}$, et adresser leurs observations au Conseil constitutionnel dans un délai de trois semaines suivant la date de transmission de la QPC.

Toutefois, force est de constater que, dans le silence des textes, la pratique des «observations» se développe aussi dans le cadre du contentieux du contrôle a priori des lois, et plus précisément des lois organiques. Dans sa décision no 2014-689 DC, le Conseil accepte en effet de prendre en compte des «observations» formulées par des parlementaires alors même que sa saisine avait été préalablement opérée par le Premier ministre sur le fondement de l'article 61, alinéa 1, de la Constitution ${ }^{17}$.

\section{Des conditions de fond à respecter}

L'année 2014 a aussi été l'occasion de rappeler le champ des dispositions susceptibles d'être contestées dans le cadre d'une QPC: seules les dispositions législatives, et leur portée effective ${ }^{18}$, peuvent faire l'objet d'un contrôle de constitutionnalité $a$ posterior $i^{19}$. Plus délicate à trancher était la question posée dans l'affaire no 2014-423 QPC: le Conseil est-il tenu d'examiner la constitutionnalité de dispositions législatives partiellement modifiées par décret, et dont les modifications (réglementaires) ne sont pas séparables des autres dispositions (législatives) ? Dans le prolongement de sa décision $\mathrm{n}^{\circ} 2013-331$ QPC du 5 juillet $2013^{20}$, il considère :

[...] qu'il revient au Conseil constitutionnel de se prononcer sur celles de ces dispositions qui revêtent une nature législative au sens de l'article 61-1 de la Constitution, en prenant en compte l'ensemble des dispositions qui lui sont renvoyées ${ }^{21}$.

8. Chapitre II bis de l'ordonnance n 58-1067 du 7 novembre 1958 (inséré par la loi organique nº 2009-1523 du 10 décembre 2009 relative à l'application de l'article 61-1 de la Constitution).

9. Art. 23-1 de l'ordonnance du 7 novembre 1958 précitée.

10. CC, déc. $\mathrm{n}^{\circ}$ 2014-440 QPC du 21 novembre 2014, M. Jean-Louis M., cons. 9.

11. Aux termes de l'art. 23-7 de l'ordonnance du 7 novembre 1958.

12. Un cinquième précédent est intervenu mais avait été rejeté par le Conseil: CC, déc. n 2012-237 QPC du 15 février 2012, M. Zafer E. En l'espèce, un même requérant avait posé deux QPC identiques à la Cour de cassation, et ce à sept jours d'intervalle. La Cour de cassation n'ayant statué que sur la première, ledit requérant saisit directement le Conseil constitutionnel. Ce dernier considéra que «compte tenu de la triple identité de requérant, de disposition législative contestée et de griefs invoqués, ainsi que de la concomitance des QPC posées », l'unique décision rendue avait eu pour effet de répondre aux deux QPC formulées (commentaire aux Cahiers du Conseil Constitutionnel de la décision n $2012-283$ QPC du 23 novembre 2012, p. 3, en ligne: http://www.conseil-constitutionnel.fr/conseil-constitutionnel/root/bank/download/2012283QPCccc_283qpc.pdf; commentaire aux Cahiers du Conseil Constitutionnel de la décision no 2014-440 QPC du 21 novembre 2014, p. 4, en ligne: http://www.conseil-constitutionnel. fr/conseil-constitutionnel/root/bank/download/2014440QPC2014440qpc_ccc.pdf).

13. CC, déc. $\mathrm{n}^{\circ} 2014-440$ QPC du 21 novembre 2014.

14. Un dessaisissement constaté par une décision juridictionnelle (CC, déc. nº 2011-206 QPC du 16 décembre 2011, M. Noël C.; CC, déc. n 2013-363 QPC du 31 janvier 2014, M. Michel P.) ou non (CC, déc. n 2012-283 QPC du 23 novembre 2012, M. Antoine de M.).

15. CC, déc. $\mathrm{n}^{\circ}$ 2011-120 ORGA du 21 juin 2011, Décision modifiant le règlement intérieur sur la procédure suivie devant le Conseil constitutionnel pour les questions prioritaires de constitutionnalité.

16. La difficulté essentielle ici réside en ce que l'article 6 du règlement du 4 février 2010, relatif à la procédure suivie devant le Conseil dans le cadre de la QPC, ne définit pas ce que recouvre la notion. Un «intérêt spécial» est généralement reconnu dès lors que le maintien ou l’abrogation de la disposition contestée traite particulièrement de la situation de l'auteur de la demande d'intervention. Exemples en 2014: CC, déc. $\mathrm{n}^{\circ}$ 2014-373 QPC du 4 avril 2014, Société Sephora; CC, déc. no 2014-374 QPC du 4 avril 2014, Société Sephora; CC, déc. n 2014-414 QPC du 26 septembre 2014, Société Assurances du Crédit mutuel; CC, déc. n 2014-419 QPC du 8 octobre 2014, Société Praxair SAS; CC, déc. nº 2014-429 QPC du 21 novembre 2014, M. Pierre T.

17. Le Conseil leur consacre à cet égard un visa: CC, déc. nº 2014-689 DC du 13 février 2014, Loi organique interdisant le cumul de fonctions exécutives locales avec le mandat de député ou de sénateur.

18. Outre la disposition législative en elle-même, le Conseil constitutionnel est en effet tenu d'examiner la constitutionnalité de la portée effective qu'une interprétation jurisprudentielle constante confère aux dispositions déférées devant lui. Cette constance est appréciée discrétionnairement au regard de deux critères: l'ancienneté, et la continuité de l'interprétation réalisée par le Conseil d'État et la Cour de cassation. Exemple en 2014 : CC, déc. no 2014-430 QPC du 21 novembre 2014, $M^{\text {me }}$ Barbara D. et autres.

19. En sont donc exclues, en bonne logique, les décisions de la Cour de cassation et du Conseil d'État (CC, déc. n 2013-363 QPC du 31 janvier 2014, cons. 11), et les ordonnances non ratifiées. Depuis la révision constitutionnelle du 23 juillet 2008, l'article 38 de la Constitution dispose que les ordonnances ne peuvent être ratifiées que de manière expresse. Dès lors, parce que cette révision ne possède pas de caractère rétroactif, seules les ratifications implicites intervenues avant le 23 juillet 2008 permettent de conférer le caractère de dispositions législatives aux dispositions implicitement ratifiées d'une ordonnance (CC, déc. $\mathrm{n}^{\circ}$ 2014-412 QPC du 19 septembre 2014, M. Laurent D., cons. 8; CC, déc. $\mathrm{n}^{\circ}$ 2014-392 QPC du 25 avril 2014, Province Sud de Nouvelle-Calédonie, cons. 10).

20. Une solution identique est retenue à propos de dispositions législatives partiellement modifiées par une ordonnance non ratifiée: CC, déc. no $2013-331$ QPC du 5 juillet 2013, Société Numericable SAS et autre, cons. 3

21. CC, déc. $n^{\circ} 2014-423$ QPC du 24 octobre 2014, M. Stéphane R. et autres, cons. 8. 
En d'autres termes, les dispositions modifiées par décret ne sont pas contrôlées, mais sont malgré tout prises en compte dans le cadre de l'examen de constitutionnalité des dispositions législatives.

Toute loi ne peut toutefois pas faire l'objet d'une QPC. Cette année, le Conseil a expressément exclu les recours portés contre les dispositions d'une loi référendaire dans le cadre du contrôle a posteriori. Cette solution existait déjà dans le cadre du contrôle a priori depuis $1962^{22}$. Saisis pour la première fois d'une telle loi dans le cadre d'une QPC, les juges de Montpensier étendent, sans grande surprise, le raisonnement retenu en $1992^{23}$.

Enfin, il convient de rappeler que seules les atteintes aux droits et libertés garantis par la Constitution peuvent être alléguées dans le cadre du contrôle a posteriori. Ainsi le Conseil a-t-il jugé cette année que le préambule de la charte de l'environnement n'instituait aucun droit ou liberté, ses dispositions ne pouvant par conséquent pas être invoquées dans le cadre d'une QPC ${ }^{24}$. Par ailleurs, il a allongé la liste des objectifs à valeur constitutionnelle (OVC) dont la méconnaissance ne peut, en elle-même, être invoquée à l'appui d'une QPC. Y figuraient déjà l'OVC d'accessibilité et d'intelligibilité de la $10 i^{25}$, ainsi que celui de bonne administration de la justice ${ }^{26}$. En 2014, le Conseil y ajoute les OVC de sauvegarde de l'ordre public ${ }^{27}$, et de bon usage des deniers publics ${ }^{28}$. Ainsi, dans le cadre d'une QPC, le grief tiré de leur méconnaissance ne peut donc pas être formulé de manière autonome, mais seulement à l'appui d'un autre grief d'inconstitutionnalité. Enfin, l'année 2014 a été l'occasion de consacrer la liberté d'enseignement ${ }^{29}$ au titre des principes faisant partie des droits et libertés que la Constitution garantit, et dont la méconnaissance peut être invoquée dans le cadre du contrôle a posteriori ${ }^{30}$.

\section{B. Le contrôle de la qualité de la loi}

Le Conseil constitutionnel veille à ce que l'œuvre du législateur soit de qualité en s'assurant, d'une part qu'il exerce sa compétence (1), et d'autre part du respect des procédures d'élaboration des lois (2).

\section{Un législateur tenu d'exercer sa propre compétence}

Depuis 1977, le Conseil constitutionnel contrôle les lois d'habilitation, par lesquelles le Parlement consent à ce que le gouvernement prenne, temporairement, des mesures qui ressortent du domaine de la loi ${ }^{31}$. Dans ce cadre, il a tôt fait de préciser que le Parlement ne peut être à l'initiative de son propre dessaisissement ${ }^{32}$. Les juges constitutionnels poursuivent ce raisonnement en 2014: à l'occasion de la décision $n^{\circ}$ 2014-700 DC, le Conseil précise que s'il n'est pas interdit aux parlementaires d'amender une disposition d'habilitation proposée par le gouvernement, un tel amendement ne peut que restreindre, et non étendre, le champ de cette dernière $^{33}$. En d'autres termes, ils ne peuvent de nouveau que consentir à, et non pas initier, un élargissement de leur dessaisissement au cours de la procédure d'adoption d'une telle disposition ou loi d'habilitation. Cette faculté est réservée aux amendements gouvernementaux.

D'autre part, le Conseil constitutionnel veille à ce que le législateur ne reporte pas sur d'autres autorités, administratives ou juridictionnelles, le soin de fixer des règles dont la détermination n'a été confiée qu'à la loi. Le grief tiré de l'« incompétence négative du législateur» renvoie à l'hypothèse dans laquelle le Parlement a méconnu l'étendue de sa compétence: soit parce que la loi reste silencieuse ${ }^{34}$, soit en raison de dispositions imprécises susceptibles de donner

22. CC, déc. n 62-20 DC du 6 novembre 1962, Loi relative à l'élection du président de la République au suffrage universel direct, adoptée par le référendum du 28 octobre 1962 , cons. 2.

23. CC, déc. n 92-313 DC du 23 septembre 1992, Loi autorisant la ratification du traité sur l'Union européenne, cons. 2; CC, déc. n² 2014-392 QPC du 25 avril 2014, cons. 7 .

24. CC, déc. $\mathrm{n}^{\circ}$ 2014-394 QPC du 7 mai 2014, Société Casuca, cons. 4 à 5.

25. Principe posé dans: CC, déc. nº 2010-4/17 QPC du 22 juillet 2010, M. Alain C. et autre, cons. 5. Mis en œuvre en 2014 dans: CC, déc. nº $2013-370$ QPC du 28 février 2014, M. Marc S. et autre, cons. 19.

26. Principe posé dans: CC, déc. $\mathrm{n}^{\circ}$ 2010-77 QPC du 10 décembre 2010, $M^{\text {me }}$ Barta $Z$., cons. 3

27. CC, déc. $\mathrm{n}^{\circ}$ 2014-422 QPC du 17 octobre 2014, Chambre syndicale des cochers chauffeurs CGT-taxis, cons. 12.

28. CC, déc. $\mathrm{n}^{\circ}$ 2014-434 QPC du 5 décembre 2014, Société de laboratoires de biologie médicale Bio Dômes Unilabs SELAS, cons. 7.

29. La liberté d'enseignement constitue un principe fondamental reconnu par les lois de la République (PFRLR): CC, déc. $\mathrm{n}^{\circ} 77-87$ DC du 23 novembre 1977, Loi complémentaire à la loi no 59-1557 du 31 décembre 1959 modifiée par la loi $n^{\circ} 71-400$ du $1^{\text {er }}$ juin 1971 et relative à la liberté de l'enseignement, cons. 2 et 3 .

30. CC, déc. $\mathrm{n}^{\circ}$ 2014-425 QPC du 14 novembre 2014, Société Mutuelle Saint-Christophe.

31. CC, déc. $n^{\circ}$ 76-72 DC du 12 janvier 1977, Loi autorisant le gouvernement à modifier par ordonnances les circonscriptions pour l'élection des membres de la chambre des députés du territoire français des Afars et des Issas.

32. Seul le gouvernement est autorisé à demander une habilitation législative: CC, déc. n²004-510 DC du 20 janvier 2005, Loi relative aux compétences du tribunal d'instance, de la juridiction de proximité et du tribunal de grande instance, cons. 28. Cette demande peut intervenir à tout moment de la procédure législative, par le biais d'un projet de loi ou un amendement à un texte en cours d'examen: CC, déc. n² 2006-534 DC du 16 mars 2006, Loi pour le retour à l'emploi et sur les droits et les devoirs des bénéficiaires de minima sociaux, cons. 5.

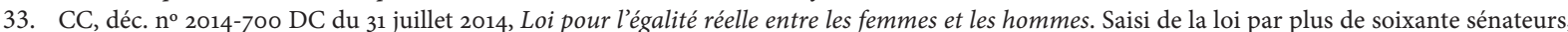
le Conseil examina d'office l'habilitation à légiférer par ordonnances résultant de l'article 74 de la loi ainsi déférée. Il formula alors une réserve d'interprétation conditionnant sa constitutionnalité (cons. 9), tendant à neutraliser une proposition de rédaction adoptée par la Commission mixte paritaire qui avait eu pour effet d'élargir le champ de l'habilitation.

34. Cette hypothèse a conduit à plusieurs censures au cours de l'année: CC, déc. no 2014-388 QPC du 11 avril 2014, Confédération générale du travail Force ouvrière et autre, cons. 6; CC, déc. n² 2014-393 QPC du 25 avril 2014, M. Angelo R., cons. 6; CC, déc. n² 2014-395 QPC du 7 mai 2014, Fédération environnement durable et autres, cons. 11. 
lieu à une pluralité d'interprétation ${ }^{35}$, soit parce que la loi renvoie intégralement les modalités d'application à des lois futures ${ }^{36}$. À noter : la portée de ce grief est davantage limitée dans le cadre du contrôle a posteriori. Dans cette hypothèse, il ne peut en effet être invoqué que dans la mesure où ladite méconnaissance affecte, par elle-même, un droit ou une liberté que la Constitution garantit ${ }^{37}$. S'il n'hésite pas à sanctionner les incompétences négatives du législateur ${ }^{38}$, le Conseil constitutionnel évite parfois de recourir à la censure des dispositions législatives. Ainsi se réfère-t-il occasionnellement à l'intention du législateur pour combler le silence d'un texte. La décision n ${ }^{\circ}$ 2014-419 QPC en constitue un bon exemple: le requérant soutenait notamment qu'en ne précisant pas les règles relatives au contentieux de la contribution au service public de l'électricité (CSPE), le législateur avait méconnu l'étendue de sa compétence. Le Conseil considère alors qu'en l'absence de disposition particulière, le législateur entend renvoyer aux règles de droit commun. Or, dans la mesure où la jurisprudence constante du Tribunal des conflits et du Conseil d'État attribue le contentieux de la contribution au service public de l'électricité à la juridiction administrative, il en déduit que les règles régissant ce contentieux sont suffisamment définies ${ }^{39}$. Une autre technique consiste à formuler des réserves, visant à neutraliser des interprétations possibles de la loi. C'est ce que le Conseil constitutionnel fait dans sa décision $n^{\circ}$ 2014-431 QPC. Étaient en cause les dispositions du paragraphe II de l'article 209 du Code général des impôts, lesquelles ne précisaient pas les conditions de délivrance d'un agrément ministériel, permettant de moduler l'assiette de l'imposition des bénéfices des sociétés dans le cadre d'opérations de restructuration. Le Conseil considère alors que le législateur n'a pas méconnu l'étendue de sa compétence affectant par elle-même le principe d'égalité devant les charges publiques, sous la réserve suivante:
Les dispositions contestées ne sauraient, sans priver de garanties légales les exigences qui résultent de l'article 13 de la Déclaration de 1789, être interprétées comme permettant à l'administration de refuser cet agrément pour un autre motif que celui tiré de ce que l'opération de restructuration en cause ne satisfait pas aux conditions fixées par la loi ${ }^{40}$.

Le recours à l'intention du législateur et aux réserves neutralisantes apparaît ici comme des techniques efficaces permettant aux juges constitutionnels de pallier l'incompétence négative du législateur, tout en évitant la censure des dispositions en cause.

\section{Le contrôle de la procédure législative}

Le respect de la procédure législative ne peut être examiné que dans le cadre du contrôle a priori des lois ${ }^{41}$. Il n'existe d'ailleurs pas une seule procédure législative en France mais bien une pluralité. À la procédure ordinaire, s'ajoutent des procédures législatives spéciales ${ }^{42}$, dont le champ d'application est contrôlé par le Conseil. Ainsi n'hésite-t-il pas à censurer les dispositions insérées à tort dans les lois de finances ${ }^{43}$ et de financement de la Sécurité sociale ${ }^{44}$. Il ne procède, par contre, qu'au simple « déclassement ", sans annulation, des dispositions insérées à tort dans une loi organique ${ }^{45}$.

Une question particulière se posa dans la décision $\mathrm{n}^{\circ}$ 2014-689 DC en ce qui concerne la procédure d'adoption des lois organiques. Rappelons qu'en cas de désaccord entre les deux assemblées, l'article 46 de la Constitution offre «le dernier mot» à l'Assemblée nationale, sauf s'il s'agit d'une «loi organique relative au Sénat». Les juges de Montpensier retiennent une conception relativement restrictive de cette exception: seules les dispositions des lois organiques concernant exclusivement le Sénat reçoivent ce label, et nécessitent donc un vote dans les

35. CC, déc. $\mathrm{n}^{\circ}$ 2014-708 DC du 29 décembre 2014, Loi de finances rectificative pour 2014, cons. 31.

36. Hypothèse plus rare, notamment sanctionnée dans: CC, déc. $\mathrm{n}^{\circ} 2004-499$ DC du 29 juillet 2004 , Loi relative à la protection des personnes physiques à l'égard des traitements de données à caractère personnel et modifiant la loi $n^{\circ} 78-17$ du 6 janvier 1978 relative à l'informatique, aux fichiers et aux libertés, cons. 12.

37. Principe posé par CC, déc. $\mathrm{n}^{\circ}$ 2012-254 QPC du 18 juin 2012, Fédération de l'énergie et des mines - Force ouvrière FNEM FO, cons. 3. Quelques innovations en la matière peuvent être soulignées ici. Cette année, le Conseil a en effet considéré que l'incompétence négative du législateur pouvait affecter la liberté de communication des pensées et des opinions (CC, déc. n²013-364 QPC du 31 janvier 2014, Coopérative GIPHAR-SOGIPHAR et autre), la liberté individuelle, la liberté d’aller et venir et le respect de la vie privée (CC, déc. nº 2013-367 QPC du 14 février 2014, Consorts L.), ainsi que le principe de participation du public (CC, déc. no 2014-395 QPC du 7 mai 2014).

38. Il n'hésite d'ailleurs pas à soulever d'office le grief: CC, déc. nº 2014-388 QPC du 11 avril 2014, cons. 2; CC, déc. nº $2014-708$ DC du 29 décembre 2014, cons. 31.

39. CC, déc. n 2014-419 QPC du 8 octobre 2014, cons. 16. (dans des conditions portant atteinte au droit de propriété, au droit à un recours juridictionnel effectif et au principe du consentement à l'impôt).

40. CC, déc. $\mathrm{n}^{\circ}$ 2014-431 QPC du 28 novembre 2014, Sociétés ING Direct NV et ING Bank NV, cons. 11.

41. Le Conseil a eu l'occasion de le rappeler cette année, qu'il s'agisse d'une loi ordinaire (CC, déc. nº 2013-370 QPC du 28 février 2014, cons. 11$)$ ou organique (CC, déc. n 2014-386 QPC du 28 mars 2014, Collectivité de Saint-Barthélemy, cons. 8).

42. Les lois constitutionnelles, lois organiques, lois autorisant la ratification d'un engagement international, lois de finances et de financement de la Sécurité sociale sont adoptées selon des procédures législatives spéciales.

43. Il censure en ce sens tout « cavalier budgétaire », c'est-à-dire toute disposition dont la présence dans une loi de finances est proscrite par l'article 34 alinéa 19 de la Constitution et l'article $1^{\text {er }}$ de la loi organique relative aux lois de finances: CC, déc. n ${ }^{\circ} 2014-707$ du 29 décembre 2014 , Loi de finances pour 2015, cons. 63; CC, déc. $\mathrm{n}^{\circ}$ 2014-708 DC du 29 décembre 2014, cons. 43.

44. Il en va de même en ce qui concerne les "cavaliers sociaux» (disposition dont la présence dans une loi de financement de la Sécurité sociale est proscrite par l'article 34 alinéa 20 de la Constitution et l'article $1^{\text {er }}$ de la loi organique relative aux lois de financement de la Sécurité sociale): CC, déc. n 2014-698 DC du 6 août 2014, cons. 20; CC, déc. n² 2014-706 DC du 18 décembre 2014, Loi de financement de la Sécurité sociale pour 2015, cons. 44

45. CC, déc. $\mathrm{n}^{\circ} 2014-689$ DC du 13 février 2014, cons. 41. 
mêmes termes par les deux assemblées ${ }^{46}$. En l'espèce, il s'agissait de la loi organique interdisant le cumul de fonctions exécutives locales avec le mandat de député ou de sénateur. Le Conseil rappela que seules les dispositions concernant l'ensemble des parlementaires, et non les sénateurs seuls, pouvaient être adoptées en lecture définitive par l'Assemblée nationale dans une rédaction différente de celle adoptée en nouvelle lecture par le Sénat. Ce n'était pas le cas de l'article 8 de la loi déférée, lequel établissait les règles régissant le remplacement des sièges vacants de parlementaires. Or les élections des députés et des sénateurs répondent à des modalités distinctes, le remplacement de leurs sièges vacants a fortiori aussi. Le Conseil en déduit que

Ces dispositions, qui, s'agissant des sénateurs élus à la représentation proportionnelle, se distinguent de celles relatives aux conditions dans lesquelles il est procédé à des élections partielles pour le remplacement des sièges vacants de députés, sont relatives au Sénat ${ }^{47}$.

Il déclara donc les dispositions du paragraphe IV de l'article 8 de la loi organique contraires à la Constitution, car adoptées selon une procédure contrevenant aux exigences de l'article 46, alinéa 4.

D'autre part, le Conseil constitutionnel a sanctionné à maintes reprises cette année l'exercice du droit d'amendement, qui ne respectait pas les exigences découlant de l'article 45 de la Constitution. Rappelons que tout amendement est recevable, au cours de la première lecture, dès lors qu'il présente un lien, même indirect, avec le texte déposé ou transmis. En ce sens, le Conseil censure en tant que «cavalier législatif» toute disposition issue d'un amendement qui ne présente aucun lien avec ledit texte, que l'amendement soit d'origine gouvernementale ou parlementaire. Au cours des lectures suivantes, le droit d'amendement est envisagé de manière nettement plus restrictive: sont, alors, seuls recevables les amendements dictés par la nécessité de respecter la Constitution, d'assurer une coordination avec d'autres textes en cours d'examen ou de rectifier une erreur matérielle, ou encore, les amendements en relation directe avec une disposition du texte restant en discussion ${ }^{48}$. Cette dernière hypothèse renvoie à la règle dite « de l'entonnoir ${ }^{49}$. Ainsi le Conseil constitutionnel n'a-t-il pas hésité cette année à censurer un cavalier législatif en première lecture ${ }^{50}$, et relever d'office à trois reprises une méconnaissance de la règle de l'entonnoir ${ }^{51}$.

\section{Le contrôle substantiel}

\section{A. Le droit de propriété}

Chaque année, le Conseil est confronté à maintes reprises au droit de propriété. L'année 2014 n'échappe pas à cette tendance, ce qui a permis aux juges de Montpensier de préciser leur jurisprudence afférente, notamment en ce qui concerne son champ d'application. Il est acquis que la protection de ce droit s'étend sur des biens d'une grande variété, le Conseil ayant reconnu que

[Depuis] 1789 et jusqu'à nos jours les finalités et les conditions d'exercice du droit de propriété ont subi une évolution caractérisée [...] par une notable extension de son champ d'application à des domaines individuels nouveaux ${ }^{52}$

La propriété intellectuelle s'est donc naturellement développée dans ce contexte d'expansion de la définition du droit de propriété. Cette nouvelle facette est reconnue comme relevant de la compétence des juridictions judiciaires. Cependant, le Conseil, avec le développement du contrôle a posteriori, est conduit à préciser les modalités de mise en œuvre de ce droit. Il n'innove néanmoins pas totalement en la matière et s'appuie même sur la jurisprudence de la Cour de cassation. Il a par exemple confirmé une jurisprudence établie de la haute cour judiciaire ${ }^{53}$ à propos de la loi du 11 avril 1910 relative à la protection du droit des auteurs en matière de reproduction des œuvres d'art ${ }^{54}$. Ainsi, dans la QPC $\mathrm{n}^{\circ}$ 2014-430, le Conseil considère que ne porte pas une atteinte disproportionnée au droit de propriété le fait que la cession du support matériel d'une œuvre emporte le droit de reproduction de celle-ci, et ce à moins que l'auteur n'émette une réserve ${ }^{55}$.

Outre le champ d'application, le Conseil s'attache plus particulièrement aux atteintes pouvant être portées à ce droit, qu'il s'agisse d'une privation (1) ou bien d'une limitation (2).

46. J.-P. Camby, «La loi organique relative au Sénat», L'actualité juridique. Droit administratif, n 3, janvier 2013, p. 160-164.

47. CC, déc. nº 2014-689 DC du 13 février 2014, cons. 4.

48. Une jurisprudence parachevée par: CC, déc. nº 2005-532 DC du 19 janvier 2006, Loi relative à la lutte contre le terrorisme et portant dispositions diverses relatives à la Sécurité et aux contrôles frontaliers, cons. 26.

49. J.-P. Camby, «Droit d'amendement et navette parlementaire: une évolution achevée», Revue du droit public et de la science politique en France et à l'Étranger, $\mathrm{n}^{\circ}$ 2, mars 2006, p. 293.

50. CC, déc. $\mathrm{n}^{\circ}$ 2014-693 DC du 25 mars 2014, cons. 29. Le Conseil a par contre jugé le moyen inopérant dans: CC, déc. n²013-687 DC du 23 janvier 2014, cons. 12.

51. CC, déc. $\mathrm{n}^{\circ}$ 2014-700 DC du 31 juillet 2014, cons. 13; CC, déc. nº 2014-698 DC du 6 août 2014, cons. 23; CC, déc. nº 2014-701 DC du 9 octobre 2014, Loi d'avenir pour l'agriculture, l'alimentation et la forêt, cons. 13 et 51.

52. CC, déc. no 81-132 DC du 16 janvier 1982, Loi de nationalisation, cons. 16.

53. Cass., $\mathrm{1}^{\mathrm{re}}$ civ., 25 mai $2005, \mathrm{n}^{\circ} \mathrm{02}-17 \cdot 305$, Bulletin civil $\mathrm{I}, \mathrm{n}^{\mathrm{o}} 23 \mathrm{O}$.

54. Loi du 9 avril 1910, JORF, 11 avril 1910, p. 3269.

55. CC, déc. $\mathrm{n}^{\circ}$ 2014-430 QPC du 21 novembre 2014, cons. 7. 


\section{La privation du droit de propriété}

Le régime du droit de propriété est strictement encadré par le Conseil en ce qui concerne les atteintes pouvant y être portées. Traditionnellement ${ }^{56}$, les juges de Montpensier distinguent entre d'une part les privations du droit de propriété prévues par l'article 17 de la DDHC, et d'autre part les conditions d'exercice de ce droit découlant de l'article 2 de la même Déclaration.

Le Conseil, en se basant sur la DDHC, considère

[...] qu'aux termes de son article 17: «La propriété étant un droit inviolable et sacré, nul ne peut en être privé, si ce n'est lorsque la nécessité publique, légalement constatée, l'exige évidemment, et sous la condition d'une juste et préalable indemnité ${ }^{57}$.

Il ressort donc de ces dispositions que la privation de propriété, pour être validée, doit réunir trois conditions: qu'elle réponde à une nécessité publique, qu'elle fasse l'objet d'une juste et préalable indemnisation, et enfin qu'il existe un recours juridictionnel contre la décision ${ }^{58}$. À la lecture de la jurisprudence constitutionnelle, il ressort une différence entre la définition constitutionnelle et civiliste de la notion de privation du droit de propriété ${ }^{59}$. Le Conseil est en effet amené à traiter de cas de privation sans les rattacher à l'article 17 de la $\mathrm{DDHC}^{60}$. Alors que le lien d'exclusivité entre le propriétaire et le bien est rompu, le Conseil ne va pas considérer cela comme une privation du droit de propriété, mais plutôt comme une condition d'exercice de ce droit ${ }^{61}$. Il juge ainsi contraires à la Constitution les dispositions de la loi du 23 juin 1941 relative au droit de rétention au profit de l'État sur les œuvres d'art devant être exportées ${ }^{62}$. Il estime en effet que le régime mis en place porte une atteinte disproportionnée au droit de propriété au regard de l'objectif d'intérêt général poursuivi de maintien sur le territoire national des objets présentant un intérêt national ${ }^{63}$.

La procédure de transfert au profit de l'État des biens mis sous scellés, prévue par le Code de procédure pénale ${ }^{64}$, est également passée entre les mains des Sages. Dans une QPC n² 2014-406, ces derniers valident le dispositif dans la mesure où l'atteinte au droit de propriété qui résulte de son application est proportionnelle à l'OVC de bonne administration de la justice et de bon emploi des deniers publics ${ }^{65}$. Point intéressant à soulever, ce même article 41-4 du Code de procédure pénale avait déjà fait l'objet d'une QPC quelques mois plus tôt ${ }^{66}$, mais concernant son alinéa 4 relatif à la procédure de destruction des biens saisis. Enfin, le Conseil a validé le droit de préemption accordé aux sociétés d'aménagement foncier et d'établissement rural (SAFER), dans le cadre d'une aliénation à titre gratuit de l'usufruit ou de la nue-propriété d'un bien, sous réserve que ce droit

[...] ne soit [pas] mis en œuvre pour des motifs qui ne se rattachent pas principalement à leur mission de favoriser l'installation, le maintien et la consolidation d'exploitations agricoles ou forestières afin que celles-ci atteignent une dimension économique viable ${ }^{67}$.

\section{La limitation du droit de propriété}

Le Conseil, dès lors qu'il écarte l'application de l'article 17 de la DDHC, va concentrer son raisonnement sur l'article 2 de la Déclaration de 1789 . Lors de son contrôle, il va ainsi vérifier si, d'une part, la mesure est justifiée par un motif d'intérêt général et, d'autre part, si la mesure est proportionnée au but visé.

Constitue un motif d'intérêt général justifiant une restriction du droit de propriété des actionnaires, selon le Conseil, la lutte contre les prises de participations occultes dans les sociétés cotées afin de renforcer la transparence des marchés et les règles assurant la loyauté entre les sociétés et leurs membres ${ }^{68}$. De même, la protection des citoyens est un motif justifiant la faculté reconnue au préfet d'interdire la construction ou la reconstruction de biens situés dans une zone qu'il délimite comme exposée à un risque naturel prévisible ${ }^{69}$. La loi ALUR ${ }^{70}$ a sans doute été la plus belle occasion pour le Conseil de mettre en application sa jurisprudence relative à l'article 2 de la DDHC. Dans sa décision $\mathrm{n}^{\circ}$ 2014-691 DC ${ }^{71}$, il a été amené à donner de nombreux exemples de motifs d'intérêt général justifiant

56. CC, déc. no 86-207 DC du 26 juin 1986, Loi autorisant le gouvernement à prendre diverses mesures d'ordre économique et social, cons. 58

57. Voir notamment: CC, déc. no 2013-369 QPC du 28 février 2014, Société Madag, cons. 8; CC, déc. no 2013-370 QPC du 28 février 2014 , cons. 12.

58. CC, déc. n 2010-87 QPC du 21 janvier 2011, M. Jacques S., cons. 3.

59. Voir notre chronique: "Chronique de jurisprudence constitutionnelle française 2012 ", Cahiers de la recherche sur les droits fondamentaux, $\mathrm{n}^{\circ} 11,2013$, p. 173

60. CC, déc. $\mathrm{n}^{\circ}$ 2014-426 QPC du 14 novembre 2014, M. Alain L.

61. CC, déc. $\mathrm{n}^{\circ}$ 2013-369 QPC du 28 février 2014, cons. 8; déc. nº 2013-370 QPC du 28 février 2014, cons. 12: « [...] qu’en l'absence de privation du droit de propriété au sens de cet article, il résulte néanmoins de l'article 2 de la Déclaration de 1789 que les atteintes portées à ce droit doivent être justifiées par un motif d'intérêt général et proportionnées à l'objectif poursuivi».

62. Loi du 23 juin 1941, JORF, 19 juillet 1941, p. 3030.

63. CC, déc. $\mathrm{n}^{\circ}$ 2014-426 QPC du 14 novembre 2014, cons. 6.

64. Code de procédure pénale, art. 41-4, al. 3

65. CC, déc. n 2014-406 QPC du 9 juillet 2014, M. Franck I., cons. 7.

66. CC, déc. $\mathrm{n}^{\circ}$ 2014-390 du 11 avril 2014, M. Antoine H. : en l'espèce, il ne s'agissait pas de la procédure de transfert de propriété au profit de l'État, mais de la possibilité offerte au procureur de la République de décider de la destruction des biens saisis.

67. CC, déc. $\mathrm{n}^{\circ}$ 2014-701 DC du 9 octobre 2014, cons. 21

68. CC, déc. n²013-369 QPC du 28 février 2014, cons. 9.

69. CC, déc. $\mathrm{n}^{\circ}$ 2014-411 QPC du 8 septembre 2014, Commune de Tarascon, cons. 14.

70. Loi n 2014-366 du 24 mars 2014 pour l'accès au logement et un urbanisme rénové, JORF, nº 0072, 26 mars 2014, p. 5809.

71. CC, déc. n 2014-691 DC du 20 mars 2014, Loi pour l'accès au logement et un urbanisme rénové 
une atteinte au droit de propriété, tels que la protection des personnes âgées ${ }^{72}$, ou encore la lutte contre la pénurie de logement ${ }^{73}$. Cependant, concernant la mesure phare de la loi, à savoir l'encadrement des loyers, le juge constitutionnel s'est montré plus mesuré en émettant une réserve sur les critères utilisés pour déterminer leur valeur ${ }^{74}$. Il a également censuré le mécanisme permettant de greffer au loyer un complément exceptionnel «pour des logements présentant des caractéristiques de localisation ou de confort exceptionnelles par leur nature et leur ampleur ${ }^{75}$.

Enfin, des conciliations entre le droit de propriété et une autre disposition constitutionnelle peuvent être apportées par le législateur. Dès lors, le Conseil se réfère aux conditions posées par l'article 2 afin de vérifier si la conciliation apportée ne porte pas une atteinte excessive au droit de propriété ${ }^{6}$.

\section{B. La liberté d'entreprendre}

En 2014, la liberté d'entreprendre s'est invitée au cœur de l'actualité politique et médiatique, notamment par la loi dite "Florange » ${ }^{77}$. L'intensité des débats politiques fut prolongée par une partie de la doctrine dans des termes à peine plus feutrés ${ }^{78}$. Cependant, la jurisprudence du Conseil constitutionnel relative à la liberté d'entreprendre n'aura pas connu de grands bouleversements en 2014 en confirmant la faible autonomie (1) et le caractère limité de cette liberté (2).

\section{Une liberté peu autonome}

Dès 1982, le Conseil constitutionnel souligna l'intervention nécessaire du législateur, cette liberté ne pouvant « exister que dans le cadre d'une réglementation instituée par la loi ${ }^{79}$. Par sa décision $n^{\circ}$ 2014-388 QPC relative au portage salarial, le Conseil soulève d'office l'incompétence négative du législateur ${ }^{80}$, qui a confié «aux partenaires sociaux la mission d'organiser le portage salarial sans fixer lui-même les principes essentiels de son régime juridique ${ }^{8_{1}}$. En confiant à une convention collective l'organisation du portage salarial, le législateur a méconnu sa compétence relative à la détermination des "principes fondamentaux des obligations civiles et commerciales ${ }^{82}$, auxquels il rattache directement les dispositions relatives aux " conditions essentielles de l'exercice d'une profession ou d'une activité économique ${ }^{83}$. Le Conseil conclut que cette méconnaissance affecte l'exercice de la liberté d'entreprendre. Le plein exercice de sa compétence par le législateur n'est toutefois pas suffisant. La qualité du travail législatif est également appréciée au regard de l'OVC d'accessibilité et d'intelligibilité de la loi. Cette nécessité fut invoquée dans la décision $\mathrm{n}^{\circ}$ 2014-692 DC relative à la loi visant à reconquérir l'économie réelle ${ }^{84}$. Les auteurs de la saisine mettaient en cause les dispositions relatives à l'obligation d'information et aux avis du comité d'entreprise lorsqu'une entreprise est visée par une offre publique d'acquisition. Selon les requérants, ce défaut d'accessibilité et d'intelligibilité de la loi portait atteinte à la liberté d'entreprendre. Néanmoins, le Conseil écarta ce grief ${ }^{85}$.

La faible autonomie de la liberté d'entreprendre est également perceptible dans de nombreuses décisions où elle n'est pas invoquée seule mais combinée avec d'autres droits et libertés. Dans la décision no 2014-691 DC, la liberté d'entreprendre est combinée à la liberté contractuelle pour apprécier une disposition imposant une clause d'exclusivité entre les "vendeurs de listes immobilières" et un propriétaire immobilier ${ }^{86}$. Le Conseil écarta ce grief. C'est essentiellement le droit de propriété qui est combiné à la liberté d'entreprendre. Le lien étroit qui unit la liberté d'entreprendre et le droit de propriété a été mis en évidence par la doctrine ${ }^{87}$, même si au regard de sa jurisprudence constante, le Conseil constitutionnel fait découler cette liberté de l'article 4 de la DDHC. Dans la décision no 2014-692 DC, la liberté d'entreprendre et le

72. CC, déc. n² 2014-691 DC du 20 mars 2014, cons. 15.

73. Ibid., cons. 41 .

74. Ibid., cons. 25

75. Ibid., cons. 26: le Conseil estime que le dispositif porte une atteinte disproportionnée au droit de propriété et à la liberté contractuelle, dans la mesure où ne sont pris en compte que les critères de la catégorie du logement et sa localisation, ce qui exclut des logements de l'application du dispositif alors que leurs caractéristiques propres seraient de nature à justifier un loyer d'un montant plus élevé.

76. CC, déc. n 2014-394 QPC du 7 mai 2014; voir N. Reboul-Mauphin, «Relations de voisinage et rapports de servitude: des métamorphoses en perspective!», Les petites affiches, n² 250, 16 décembre 2014, p. 7-10; M. Mekki, «"Écologisation” du droit civil des biens à l'aune de la Charte de l'environnement», La semaine juridique, éd. $G$, no 26, 30 juin 2014, p. 1293-1296.

77. Loi $\mathrm{n}^{\circ}$ 2014-384 du 29 mars 2014 visant à reconquérir l'économie réelle, JORF, $\mathrm{n}^{\circ} 77,1^{\mathrm{er}}$ avril 2014, p. 6227.

78. Voir J.-P. Chazal, «Propriété et entreprise: le Conseil constitutionnel, le droit et la démocratie», Dalloz, 2014, p. 1101; L. D’Avout, «La liberté d'entreprendre au bûcher?», Dalloz, 2014, p. 1287.

79. CC, déc. no 82-181 DC du 27 juillet 1982, Loi sur la communication audiovisuelle.

80. Le Conseil a déjà eu l'occasion de soulever d'office l'incompétence négative du législateur affectant la liberté d'entreprendre dans une QPC: CC, déc. $\mathrm{n}^{\mathrm{o}}$ 2013-336 QPC du $1^{\mathrm{er}}$ août 2013, Société Natixis Asset Management.

81. CC, déc. $\mathrm{n}^{\circ}$ 2014-388 QPC du 11 avril 2014, cons. 2.

82. Ibid., cons. 5 .

83. Ibid.

84. CC, déc. $\mathrm{n}^{\circ}$ 2014-692 DC du 27 mars 2014, Loi visant à reconquérir l'économie réelle.

85. Ibid., cons. 33 .

86. CC, déc. nº 2014-691 DC du 20 mars 2014, cons. 46 à 59.

87. Voir G. Carcassonne, «La liberté d'entreprendre», Revue Lamy droit des affaires, nº 55 (nº spécial), 2010, p. 45; V. Delvolvé, La liberté d'entreprendre, thèse de doctorat sous la direction d'Y. Gaudemet, université Paris II, 2002, 2 vol.; J.-L. Mestre, "Le Conseil constitutionnel, la liberté d'entreprendre et la propriété», Dalloz, 1984, p. 1. 
droit de propriété sont combinés pour apprécier les dispositions relatives à l'obligation adressée aux entreprises de rechercher un repreneur. Ces dispositions imposaient notamment à l'entreprise, qui envisage de fermer un établissement, d'accepter une "offre de reprise sérieuse». Un refus de cession ne pouvait être motivé que par « un motif légitime, à savoir la mise en péril de la poursuite de l'ensemble de l'activité de l'entreprise». Le Conseil censure une partie de ces dispositions, car

L'obligation d'accepter une offre de reprise sérieuse en l'absence de motif légitime et la compétence confiée à la juridiction commerciale pour réprimer la violation de cette obligation font peser sur les choix économiques de l'entreprise, notamment relatifs à l'aliénation de certains biens, et sur sa gestion des contraintes qui portent tant au droit de propriété qu'à la liberté d'entreprendre une atteinte manifestement disproportionnée au regard de l'objectif poursuivi ${ }^{88}$

Cette décision a également permis au Conseil constitutionnel de confirmer sa jurisprudence relative au pouvoir d'appréciation du chef d'entreprise auquel ne doit pas se substituer le juge ${ }^{89}$. Toujours dans cette décision, le Conseil a dû apprécier les dispositions relatives aux conditions d'attribution d'actions gratuites au regard de la liberté d'entreprendre et du droit de propriété, sans toutefois les censurer. La même combinaison est à constater dans la décision $n^{\circ}$ 2014-701 DC relative à la loi d'avenir pour l'agriculture, l'alimentation et la forêt ${ }^{90}$. Le Conseil a été amené à statuer sur la constitutionnalité de nouvelles dispositions du Code rural et de la pêche maritime portant sur les autorisations administratives d'exploiter. Il a ainsi censuré la soumission des agrandissements d'exploitation agricole à un régime d'autorisation, tels que définis comme «toute prise de participation, quelle que soit son importance». Ont été également censurées les dispositions qui

[...] ont pour effet d'interdire aux sociétés d'exploitation agricole [...] d'ajuster le volume de leur main-d'œuvre en fonction des besoins de leur exploitation compte tenu des fluctuations de l'activité économique, sauf à s'exposer au risque de voir leur autorisation d'exploiter remise en cause $^{91}$.
Enfin, une dernière illustration de la combinaison de la liberté d'entreprendre et du droit de propriété est à relever dans la décision $\mathrm{n}^{0}$ 2014-375 QPC $^{92}$.

\section{Une liberté limitée}

Dans plusieurs décisions rendues cette année, le Conseil maintient sa jurisprudence:

Il est loisible au législateur d'apporter à la liberté d'entreprendre qui découle de l'article 4 de la Déclaration de 1789 des limitations liées à des exigences constitutionnelles ou justifiées par l'intérêt général, à la condition qu'il n'en résulte pas d'atteintes disproportionnées au regard de l'objectif poursuivi ${ }^{93}$.

Parmi ces exigences constitutionnelles, la mise en œuvre par le législateur de la première phrase du cinquième alinéa du préambule de la Constitution de $1946^{94}$, c'est-à-dire le droit pour chacun d'obtenir un emploi, a permis de porter des atteintes non manifestement disproportionnées à la liberté d'entreprendre. L'OVC de sauvegarde de l'ordre public a été de nouveau utilisé pour justifier le monopole du droit de stationner et de circuler en quête de clients accordé aux taxis ${ }^{95}$.

À côté des exigences constitutionnelles, le législateur peut limiter la liberté d'entreprendre par des objectifs d’intérêt général. Ainsi, relève de l’intérêt général l'objectif

$[\ldots]$ de favoriser l'installation d'agriculteurs, [...] de consolider ou maintenir les exploitations, promouvoir le développement des systèmes de production et de maintenir une agriculture diversifiée, riche en emplois et génératrice de valeur ajoutée ${ }^{96}$,

ou la préservation de l'emploi agricole ${ }^{97}$. La lutte contre «l'antibiorésistance» en médecine vétérinaire est également un motif d'intérêt général pouvant limiter la liberté d'entreprendre. Les observations du gouvernement faisaient toutefois reposer la lutte contre «l'antibiorésistance» sur le onzième alinéa du préambule de la Constitution de 1946 relatif à la protection de la santé ${ }^{98}$. Ce choix n'est pas sans conséquence, car l'intensité du contrôle opéré par le Conseil varie en fonction de la nature de la limitation.

88. CC, déc. $\mathrm{n}^{\mathrm{o}}$ 2014-692 DC du 27 mars 2014, cons. 21.

89. Ibid., cons. 20. Le Conseil confirme sa jurisprudence: CC, déc. nº 2001-455 DC du 12 janvier 2002, Loi de modernisation sociale, cons. 49.

90. CC, déc. $\mathrm{n}^{\circ}$ 2014-701 DC du 9 octobre 2014.

91. Ibid., cons. 42.

92. CC, déc. $\mathrm{n}^{\circ}$ 2014-375 QPC du 21 mars 2014, M. Bertrand L. et autres. Les dispositions contestées relatives à la saisie de navires ne donnaient pas satisfaction aux exigences procédurales découlant de l'article 16 DDHC, privant ainsi «de garanties légales la protection constitutionnelle de la liberté d'entreprendre et du droit de propriété» (cons. 14)

93. CC, déc. n 2013-686 DC du 23 janvier 2014, Loi relative aux modalités de mise en cuvre des conventions conclues entre les organismes d'assurances maladie complémentaire et les professionnels de santé, cons. 11; CC, déc. n² 2014-375 QPC du 21 mars 2014; CC, déc. $n^{\circ} 2014-692$ DC du 27 mars 2014, cons. 7; CC, déc. no 2014-422 QPC du 17 octobre 2014, cons. 8.

94. CC, déc. $\mathrm{n}^{\circ}$ 2014-692 DC du 27 mars 2014, cons. 8. L'utilisation du cinquième alinéa du préambule de la Constitution de 1946 n'est pas nouvelle: CC, déc. nº 98-401 DC du 10 juin 1998, Loi d'orientation et d'incitation relative à la réduction du temps de travail, cons. 26.

95. CC, déc. $\mathrm{n}^{\circ}$ 2014-422 QPC du 17 octobre 2014, cons. 10. Le Conseil confirme sa jurisprudence relative aux taxis: CC, déc. $\mathrm{n}^{\circ}$ 2013-318 QPC du 7 juillet 2013, Mohamed T., cons. 13 et 14 .

96. CC, déc. n 2014-701 DC du 9 octobre 2014, cons. 36.

97. Ibid., cons. 41.

98. Ibid., cons. 46 . 
Le contrôle est restreint lorsque l'atteinte à la liberté d'entreprendre relève d'une exigence constitutionnelle: le Conseil ne sanctionne alors que les atteintes manifestement disproportionnées. En revanche, le contrôle devient normal lorsque l'atteinte est un objectif d'intérêt général: le Conseil sanctionne toute atteinte disproportionnée ${ }^{99}$. Ainsi, dans sa décision no 2014-692 DC, le Conseil apprécie le caractère manifestement disproportionné d'une atteinte fondée sur le préambule de la Constitution de $1946^{100}$.

Mais la décision no 2014-701 DC sème le doute quant à l'intensité du contrôle opéré par le Conseil. Certaines dispositions poursuivant un intérêt général (l'installation des agriculteurs) «ont porté au droit de propriété et à la liberté d'entreprendre une atteinte disproportionnée ${ }^{101}$. En revanche, d'autres dispositions poursuivant également un intérêt général (la préservation de l'emploi agricole) constituent «une atteinte manifestement disproportionnée ${ }^{102}$. Enfin, la décision n ${ }^{\circ}$ 2014-422 QPC confirme le doute sur la cohérence du Conseil dans l'intensité de son contrôle. Alors que l'OVC de sauvegarde de l'ordre public limite la liberté d'entreprendre des sociétés de "voitures de tourisme avec chauffeur», le Conseil constate simplement que les dispositions en cause ne portent pas à cette liberté «une atteinte disproportionnée ${ }^{103}$. Dans une QPC similaire, le Conseil constatait que les mêmes mesures d'ordre public «apportent à la liberté d'entreprendre des restrictions qui ne sont pas manifestement disproportionnées ${ }^{104}$.

\section{L'article 16 de la Déclaration des droits de l'homme et du citoyen}

Comme l'explique Régis Fraisse,

Pendant près de 35 ans, rue de Montpensier, évoquer l'article 16, c'était se référer à l'article 16 de la Constitution sur les circonstances exceptionnelles, c'était se référer à l'expérience traumatisante de $1961^{105}$.
Ce n'est assurément plus le cas aujourd'hui comme le démontre le nombre important de décisions rendues par le Conseil faisant appel, non plus à l'article 16 de la Constitution, mais à l'article 16 de la DDHC qui dispose que «Toute Société dans laquelle la garantie des Droits n'est pas assurée, ni la séparation des Pouvoirs déterminée, n’a point de Constitution ${ }^{106}$. Si de l'article 16 découle évidemment le principe de séparation des pouvoirs ${ }^{107}$, trouvent également leur source dans cette disposition le principe d'indépendance et d'impartialité des juridictions (1), le droit au recours (2), ou encore les droits de la défense (3).

\section{L'indépendance et l'impartialité des juridictions}

Le Conseil constitutionnel, dans sa décision du 11 décembre 2014, s'est prononcé sur la loi relative à la désignation des conseillers prud'homaux ${ }^{108}$. Alors que ces derniers étaient auparavant élus, ils seront désormais désignés en fonction del'audience des organisations syndicales de salariés et des organisations professionnelles d'employeurs. C'est sans surprise que cette disposition issue d'une loi d'habilitation a été déclarée conforme ${ }^{109}$, mais le Conseil sera peut-être amené à se prononcer de nouveau sur cette question ${ }^{110}$. Le principe d'impartialité était également invoqué, aux côtés de celui de la séparation des autorités de poursuite et de jugement, dans la décision portant sur la contrainte pénale. Les dispositions contestées prévoient que c'est le juge qui fixe cette peine, mais «si la juridiction de jugement ne l'a pas fait, le juge de l'application des peines fixe les obligations et interdictions particulières auxquelles est astreint le condamné ${ }^{111}$. En dépit du rôle étendu du juge de l'application des peines ${ }^{112}$, la décision du Conseil est assez expéditive sur ce point ${ }^{113}$. Selon lui, les principes de l'article 16 ne sont pas méconnus ${ }^{114}$.

En 2014, le Conseil a été amené à se prononcer à plusieurs reprises sur des dispositions relatives à la saisine d'office des juridictions commerciales. Certains ont pensé à ce sujet que le Conseil faisait «souffler un vent de panique

99. Commentaire aux Cahiers du Conseil constitutionnel de la décision n 2014-701 DC du 9 octobre 2014, p. 14, en ligne: http://www.conseil-constitutionnel.fr/conseil-constitutionnel/root/bank/download/2014701DC2014701dc_ccc.pdf; R. Fraisse, «La question prioritaire de constitutionnalité et la liberté d'entreprendre», Revue juridique de l'économie publique, août-septembre 2011, p. 3.

100. CC, déc. n 2014-692 DC du 27 mars 2014, cons. 11 et 21.

101. CC, déc. no 2014-701 DC du 9 octobre 2014, cons. 37.

102. Ibid., cons. 43 (nous soulignons).

103. CC, déc. $\mathrm{n}^{\mathrm{o}}$ 2014-422 QPC du 17 octobre 2014, cons. 10.

104. CC, déc. $\mathrm{n}^{\circ}$ 2013-318 QPC du 7 juin 2013, cons. 14 (nous soulignons).

105. R. Fraisse, "L'article 16 de la Déclaration, clef de voûte des droits et libertés ", Les nouveaux cahiers du Conseil constitutionnel, nº 44, juin 2014, p. 9.

106. Ce principe, invoqué environ soixante-dix fois jusqu'en 2010, a été invoqué plus de cent cinquante fois entre 2010 et 2014 (ibid., p. 14).

107. La séparation des pouvoirs a été mobilisée à plusieurs reprises dans la décision CC, déc. n²014-703 DC du 19 novembre 2014, Loi organique portant application de l'article 68 de la Constitution.

108. CC, déc. $\mathrm{n}^{\circ}$ 2014-704 DC du 11 décembre 2014, Loi relative à la désignation des conseillers prud'hommes.

109. Ibid., cons. 17: «en lui-même, le principe [...] n'est pas contraire au principe d'indépendance et d'impartialité des juridictions».

110. Voir V. Orif, «L'adieu aux élections prud'homales», Lexbase Hebdo édition sociale, n 596, 8 janvier 2015.

111. Commentaire aux Cahiers du Conseil constitutionnel de la décision no 2014-696 DC du 7 août 2014, p. 2, en ligne: http://www.conseil-constitutionnel. $\mathrm{fr} /$ conseil-constitutionnel/root/bank/download/2014696DCccc_696dc.pdf.

112. C'est lui notamment qui va assurer «le suivi, le contrôle et les adaptations, qui saisit le président du tribunal aux fins de prononcer l'emprisonnement en cas de non-respect de la mesure de contrainte et qui peut le placer en détention provisoire» (CC, déc. n $2014-696$ DC du 7 août 2014 , Loi relative à l'individualisation des peines et renforçant l'efficacité des sanctions pénales, cons. 19).

113. Voir A. Seïd Algadi, «La loi "Taubira” ou la nouvelle approche de la sanction pénale. Questions à Monsieur Pierre Bricard, ancien magistrat», Lexbase Hebdo édition privée, $\mathrm{n}^{\circ}$ 582, 11 septembre 2014.

114. Ibid. 
sur les tribunaux de commerce français $»^{115}$. En effet, deux dispositions relatives à la possibilité pour les juridictions commerciales de «se saisir d'office afin de prononcer la liquidation judiciaire ou la résolution d'un plan de sauvegarde ou de redressement judiciaire d'une entreprise en difficulté ${ }^{116}$ ont été censurées ${ }^{117}$. À cette occasion, le Conseil a rappelé son considérant de principe issu de sa décision du 7 décembre $2012^{118}$. Il suit alors un raisonnement en trois temps ${ }^{119}$. Si, en principe, « une juridiction ne saurait disposer de la faculté d'introduire spontanément une instance au terme de laquelle elle prononce une décision revêtue de l'autorité de chose jugée », «la Constitution ne confère pas à cette interdiction un caractère général et absolu ${ }^{120}$. En effet, la loi peut prévoir des exceptions, mais uniquement «lorsque la procédure n'a pas pour objet le prononcé de sanctions ayant le caractère d'une punition ${ }^{121}$. Dès lors, la saisine sera admise si elle est fondée sur un motif d'intérêt général et que des garanties propres à assurer le respect du principe d'impartialité sont prévues. En l'espèce, les deux dispositions étaient bien fondées sur un motif d'intérêt général, notamment celui d' «éviter l'aggravation irrémédiable de la situation de l'entreprise ${ }^{122}$. C'est donc la seconde condition qui a fait défaut, le Conseil considérant qu'aucune garantie légale n'avait été fixée pour s'assurer que «le tribunal ne préjuge pas sa position ${ }^{123}$. Selon Damien Fallon, «Il est cependant peu probable que cette solution fasse effectivement souffler "un vent de panique sur les tribunaux de commerce français" " puisque «la censure de l'autosaisine ne s'applique qu'à l'occasion de l'ouverture d'une nouvelle instance ${ }^{124}$. Il semble donc que le raisonnement du Conseil soit plutôt subtil ${ }^{125}$ : dans sa décision du 6 juin
2014, il déclare qu'une disposition législative permettant au tribunal de commerce de se saisir d'office pour prononcer la liquidation judiciaire lors de la période d'observation du redressement judiciaire est conforme à l'article $16^{126}$. Il faut donc distinguer entre la «saisine d'office pour ouverture d'instance» et la «faculté d'exercer d'office un pouvoir au sein d'une instance ${ }^{127}$, cette dernière possibilité ne donnant alors pas lieu dans cette décision à une censure.

\section{Le droit à un recours juridictionnel effectif}

Il résulte également de l'article 16 qu'il «ne doit pas être porté d'atteintes substantielles au droit des personnes intéressées d'exercer un recours effectif devant une juridiction ${ }^{128}$. Le Conseil s'est prononcé cette année sur la question des validations législatives. Il s'agit de l'hypothèse dans laquelle le législateur "valide» un acte administratif dont l'illégalité a été reconnue par le juge administratif, entraînant ainsi une limitation importante du droit au recours. De manière classique, le Conseil soumet cette possibilité à cinq conditions ${ }^{129}$, en tête desquelles figure l'intérêt général. Sur ce point, le Conseil a innové cette année en modifiant son considérant de principe : à l'exigence d'un «but d'intérêt général suffisant», il soustrait celle de «motif impérieux d'intérêt général ${ }^{130}$. Dans cette décision relative au versement transport, correspond à un tel motif la volonté par le législateur de «mettre un terme à des années de contentieux» ou encore d' «éviter une multiplication des réclamations ${ }^{131}$. Selon le commentaire aux Cahiers du Conseil constitutionnel, le Conseil entend renforcer son exigence et ainsi, s'aligner sur la jurisprudence de la Cour européenne des droits de l'homme ${ }^{132}$. Si

115. S. Robineau, «Le Conseil constitutionnel fait souffler un vent de panique sur les tribunaux de commerce français», Les Échos, 9 mars 2014 , cité par D. Fallon, «Saisine d'office du tribunal de commerce: rien de nouveau sous le soleil de Montpensier», Gazette du palais, n 101-102, 12 avril 2014, p. 933.

116. D. Fallon, «Saisine d'office du tribunal de commerce...», p. 931.

117. CC, déc. $\mathrm{n}^{\circ}$ 2013-368 QPC du 7 mars 2014, Société Nouvelle d'exploitation Sthrau hôtel [Saisine d'office du tribunal pour l'ouverture de la procédure de liquidation judiciaire] et CC, déc. no 2013-372 QPC du 7 mars 2014, M. Marc V. [Saisine d'office du tribunal pour la résolution d'un plan de sauvegarde ou de redressement judiciaire].

118. CC, déc. $\mathrm{n}^{\circ}$ 2012-286 QPC du 7 décembre 2012, Société Pyrénées services et autres [Saisine d'office du tribunal pour l'ouverture de la procédure de redressement judiciaire], cons. 3 .

119. Commentaire aux Cahiers du Conseil constitutionnel de la décision no 2013-368 QPC du 7 mars 2014, p. 4, en ligne: http://www.conseil-constitutionnel. fr/conseil-constitutionnel/root/bank/download/2013368QPCccc_368qpc.pdf.

120. CC, déc. $\mathrm{n}^{\circ}$ 2013-372 QPC du 7 mars 2014, cons. 4.

121. Ibid.

122. Ibid., cons. 9 et CC, déc. $\mathrm{n}^{\circ}$ 2013-368 QPC du 7 mars 2014, cons. 6.

123. CC, déc. n 2013-372 QPC du 7 mars 2014, cons. 10 et CC, déc. nº 2013-368 QPC du 7 mars 2014, cons. 7.

124. Il s'agit en effet d' " une nouvelle instance distincte de celle à l'issue de laquelle le plan de sauvegarde ou le plan de redressement a été arrêté » (CC, déc. n 2013-372 QPC du 7 mars 2014, cons. 8); voir D. Fallon, «Saisine d'office du tribunal de commerce...», p. 933.

125. Voir L. C. Henry, «Les subtilités de la saisine d'office et les décisions du Conseil constitutionnel», Revue des sociétés, n 9 , septembre 2014 , p. 528.

126. CC, déc. no 2014-399 QPC du 6 juin 2014, Société Beverage and Restauration Organisation SA [Liquidation judiciaire ou cessation partielle de l'activité prononcée d'office pendant la période d'observation du redressement judiciaire].

127. J. Théron, «La constitutionnalité des pouvoirs exercés d'office par le tribunal au sein d'une instance», Chronique de jurisprudence de procédure civile, Gazette du palais, n² 252, 9 septembre 2014, p. 3102.

128. Par exemple CC, déc. nº 2013-363 QPC du 31 janvier 2014, cons. 4.

129. Par exemple, CC, déc. no 2011-224 QPC du 24 février 2012, Coordination pour la sauvegarde du bois de Boulogne [Validation législative de permis de construire], cons. 4 .

130. CC, déc. $\mathrm{n}^{\circ}$ 2013-366 QPC du 14 février 2014, SELARL PJA, ès qualités de liquidateur de la société Maflow France [Validation législative des délibérations des syndicats mixtes instituant le «versement transport»], cons. 3 .

131. Ibid., cons. 6 .

132. Commentaire aux Cahiers du Conseil constitutionnel de la décision n² 2013-366 du 14 février 2014, p. 13, en ligne: http://www.conseil-constitutionnel. fr/conseil-constitutionnel/root/bank/download/2013366QPCccc_366qpc.pdf. 
cette modification d'ordre terminologique ne devrait pas entraîner de réel changement, il n'est pas certain qu'elle entraîne un alignement total de la jurisprudence de Montpensier sur celle de Strasbourg ${ }^{133}$. En témoigne la décision rendue sur la loi relative à la sécurisation des contrats de prêts structurés souscrits par les personnes morales de droit public, plus connus sous le nom d' «emprunts toxiques ${ }^{134}$. Le Conseil se fonde ici sur le risque financier alors que la Cour EDH considère « qu'en principe le seul intérêt financier de l'État ne permet pas de justifier l'intervention rétroactive d'une loi de validation ${ }^{135}$.

L'influence de la Cour EDH est également palpable dans d'autres décisions relatives au droit au recours. C'est le cas de la décision du 13 juin 2014 qui déclare contraire à la Constitution l'article 380-11, alinéa 5, du Code de procédure pénale qui prévoit que lorsqu'un accusé fait appel de sa condamnation, mais prend la fuite et n'est pas présent lors de l'audience, la condamnation rendue devient définitive ${ }^{136}$. Le 7 novembre 2014, le Conseil assortit sa décision de conformité de l'article 5, alinéa 3, de la loi du $1^{\text {er }}$ juillet 1901, d'une réserve d'interprétation, s'alignant ainsi sur la jurisprudence européenne ${ }^{137}$. Cette disposition prévoit que «Lorsque l'association aura son siège social à l'étranger, la déclaration préalable [...] sera faite à la préfecture du département où est situé le siège de son principal établissement ${ }^{138}$. Le Conseil a donc utilement précisé que ces dispositions

[...] n'ont pas pour objet et ne sauraient, sans porter une atteinte injustifiée au droit d'exercer un recours juridictionnel effectif, être interprétées comme privant les associations ayant leur siège à l'étranger [...] de la qualité pour agir devant les juridictions françaises ${ }^{139}$,

leur retirant ainsi leur «venin d'inconstitutionnalité » ${ }^{140}$.

Dans la décision du 31 janvier 2014, a été déclaré conforme à la Constitution, et notamment au droit au recours, le troisième alinéa de l'article 497 du Code de procédure pénale. Celui-ci prévoit qu'en matière correctionnelle, lorsque le ministère public décide de ne pas faire appel d'une relaxe, la partie civile ne peut alors faire qu'une demande de dommages-intérêts ${ }^{141}$. Cette décision pouvait apparaître contradictoire avec une autre rendue en $2010^{142}$, mais comme le relève Antoine Botton:

[...] l'article 575 du code de procédure pénale cantonnait la faculté de pourvoi en cassation de la partie lésée avant toute instance au fond, tandis que l'article $497,3^{\circ}$, limite son droit d'appel après la survenance, par hypothèse, d'un premier jugement sur le fond de l'affaire ${ }^{143}$.

Ne porte pas non plus atteinte au droit au recours l'article L. 173-12 du Code de l'environnement sur la transaction pénale, le Conseil considérant que

[...] les dispositions contestées ne font pas obstacle au droit des victimes, avisées de la procédure par le procureur de la République [...], d'agir pour demander la réparation de leur préjudice devant les juridictions civiles ainsi que [...] devant les juridictions répressives ${ }^{144}$.

Enfin, dans trois décisions de censure ${ }^{145}$, le Conseil a été amené à se prononcer sur «la portée de l'exigence constitutionnelle résultant du droit à recours, en matière de contestation des actes d'investigation en cours d'enquête ${ }^{146}$. Il a notamment considéré, dans sa décision du 4 avril 2014 portant sur le travail dissimulé, qu'

[...] en l'absence de mise en œuvre de l'action publique conduisant à la mise en cause d'une personne intéressée par une visite domiciliaire, une perquisition ou une saisie autorisées en application des dispositions contestées, aucune voie de droit ne permet à cette personne de contester l'autorisation donnée par le président du TGI ou son délégué et la régularité des opérations ${ }^{147}$.

Cette décision, attendue au regard de celle rendue quelques jours plus tôt à propos des saisies conservatoires

133. Voir J. Roux, «Du "but d’intérêt général suffisant” au "motif impérieux d’intérêt général”: les mots et les choses», L'actualité juridique. Droit administratif, $\mathrm{n}^{\circ}$ 21, juin 2014, p. 1208.

134. CC, déc. $\mathrm{n}^{\circ}$ 2014-695 DC du 24 juillet 2014, Loi relative à la sécurisation des contrats de prêts structurés souscrits par les personnes morales de droit public; voir A. Roblot-Troizier, «Validation législative: le Conseil constitutionnel se soumet pour renforcer l'autorité de sa jurisprudence», Chronique de jurisprudence - droit administratif et droit constitutionnel, Revue française de droit administratif, $\mathrm{n}^{\circ} 3$, mai-juin $2014, \mathrm{p} .589$.

135. Cour EDH, 25 novembre 2010, Lilly France c. France, no 20429/07, \$51.

136. CC, déc. $\mathrm{n}^{\circ}$ 2014-403 QPC du 13 juin 2014, M. Laurent L. [Caducité de l'appel de l'accusé en fuite]; voir commentaire de la décision aux Cahiers du Conseil constitutionnel, p. 6, en ligne: http://www.conseil-constitutionnel.fr/conseil-constitutionnel/root/bank/download/2014403QPCccc_403qpc.pdf

137. Cour EDH, 15 janvier 2009, Ligue du monde islamique et organisation islamique mondiale du secours islamique c. France, $\mathrm{n}^{\circ} 36497 / 05$ et $37172 / 05, \$ 58$.

138. CC, déc. $\mathrm{n}^{\circ}$ 2014-424 QPC du 7 novembre 2014, Association Mouvement raëlien international [Capacité juridique des associations ayant leur siège social à l'étranger], cons. 1.

139. Ibid., cons. 7 .

140. S. Slama, «Réserve d'interprétation pour garantir le droit d'ester en justice aux associations ayant leur siège social à l'étranger sans établissement en France», Lettre «Actualités Droits-Libertés» de La revue des droits de l'homme, 2 décembre 2014, en ligne: https://revdh.revues.org/1022.

141. CC, déc. nº 2013-363 QPC du 31 janvier 2014.

142. CC, déc. $\mathrm{n}^{\circ}$ 2010-15/23 QPC du 23 juillet 2010, Région Languedoc-Roussillon et autres [Article 575 du Code de procédure pénale].

143. A. Botton, "Constitutionnalité de l'appel limité des parties civiles: le sérieux des questions en question", Dalloz, 2014 , p. 653.

144. CC, déc. no $\mathrm{n}^{\circ}$ 2014-416 QPC du 26 septembre 2014, Association France Nature Environnement [Transaction pénale sur l'action publique en matière environnementale], cons. 10; voir H. Hoepffner, «La transaction pénale en matière environnementale: le clair-obscur de la décision du Conseil constitutionnel ", Chronique de droit public, Les nouveaux cahiers du Conseil constitutionnel, $\mathrm{n}^{\circ}$ 46, janvier 2015, p. $161-176$.

145. CC, déc. no 2014-375 et autres QPC du 21 mars 2014; CC, déc. n 2014-387 QPC du 4 avril 2014, M. Jacques J. [Visites domiciliaires, perquisitions et saisies dans les lieux de travail]; CC, déc. $\mathrm{n}^{\circ} 2014-390$ QPC du 11 avril 2014.

146. Commentaire aux Cahiers du Conseil constitutionnel de la décision nº 2014-387 QPC du 4 avril 2014, p. 10, en ligne: http://www.conseil-constitutionnel.fr/conseil-constitutionnel/root/bank/download/2014387QPCcc__387qpc.pdf.

147. CC, déc. nº 2014-387 QPC du 4 avril 2014, cons. 7. 
opérées dans le cadre d'infractions maritimes ${ }^{148}$, n'en est pas moins importante car elle

[...] ouvre ainsi la voie à une contestation de la constitutionnalité de l'ensemble des articles du Code de procédure pénale qui autorise les forces de police à accomplir, en enquête préliminaire ou de flagrance, les gardes à vue, perquisitions et saisies ${ }^{149}$.

\section{Le respect des droits de la défense}

L'article 16 comprend aussi le respect des droits de la défense ${ }^{150}$. De cet article 16, et de l'article 6 de la DDHC, découle le principe d'égalité devant la justice. Dans sa décision du 31 janvier 2014, le Conseil a considéré que «l'interdiction faite à la partie civile d'appeler seule d'un jugement correctionnel dans ses dispositions statuant au fond sur l'action publique, ne méconnaît pas le principe d'égalité devant la justice » ${ }^{151}$. En effet, selon le Conseil, la partie civile et le ministère public ne sont pas «dans une situation identique ${ }^{152}$. Si cette solution semble logique, elle est néanmoins discutable, notamment au regard du «caractère ambigu du rôle tenu par la victime au sein du procès pénal ${ }^{153}$.

Le Conseil s'est prononcé à plusieurs reprises cette année sur la conformité de dispositions relatives à la criminalité et à la délinquance organisée au regard du respect des droits de la défense. D'abord, dans sa décision du 9 octobre, le Conseil, s'inscrivant dans le prolongement de sa jurisprudence antérieure ${ }^{154}$, a déclaré contraire à la Constitution des dispositions qui permettent de recourir à une prolongation exceptionnelle de la garde à vue en cas de délit d'escroquerie en bande organisée ${ }^{155}$. En effet, «même lorsqu'il est commis en bande organisée, le délit d'escroquerie n'est pas susceptible de porter atteinte en lui-même à la sécurité, à la dignité ou à la vie des per- sonnes » ${ }^{156}$, l'intervention de la loi du 27 mai 2014 ne mettant pas fin à l'inconstitutionnalité. Toutefois, amené à se prononcer plus particulièrement sur la constitutionnalité du report de l'intervention de l'avocat ${ }^{157}$, «Si la gravité et / ou la complexité d'une infraction commise en bande organisée justifie une garde à vue dérogatoire», le Conseil «prend soin, pour valider l'intervention reportée de l'avocat, de mettre en exergue les garanties législatives qui l'entourent $»^{158}$. Enfin, le Conseil a validé l'essentiel de la loi relative à la géolocalisation, mais a prononcé deux réserves d'interprétation ${ }^{159}$ et une censure, s'agissant du dossier de la procédure. En effet, il est prévu que certains éléments du dossier puissent être gardés secrets. L'article 230-42 du Code de procédure pénale interdisait, sauf exception, de se fonder sur le seul fondement de ce dossier «secret» pour fonder une condamnation. C'est donc sur ce point que la décision du Conseil attire tout particulièrement notre attention puisque la censure porte sur le mot «seul» : ainsi,

[...] le conseil met à bas tout le dispositif législatif de géolocalisation en matière de criminalité organisée car il y a fort à parier que, dans la plupart des cas, les informateurs, témoins ou citoyens que le dossier distinct visait à protéger ne souhaiteront certainement pas, par peur des représailles, que ce dernier soit joint à la procédure ${ }^{160}$.

\section{Le principe d'égalité}

«Dresser maintenant la liste des matières auxquelles le principe d'égalité est opposable, il faudrait être Prévert! $\gg^{161}$. Le principe d'égalité est, en effet, le principe constitutionnel qui est le plus souvent invoqué devant le Conseil constitutionnel ${ }^{162}$.

On l'appelle "principe-gigogne ${ }^{163}$ : en effet les cas auxquels il trouve à s'appliquer sont multiples et soumis

148. CC, déc. n 2014-375 et autres QPC du 21 mars 2014

149. D. Rebut, «Juge constitutionnel. Séisme sur la procédure pénale», La semaine juridique, éd. G, $\mathrm{n}^{\circ} 20,19$ mai 2014 , doctr. 598.

150. CC, déc. $\mathrm{n}^{\circ}$ 2006-540 DC du 27 juillet 2006, Loi relative au droit d'auteur et aux droits voisins dans la société de l'information, cons. 11.

151. CC, déc. n 2013-363 QPC du 31 janvier 2014, cons. 8.

152. Le Conseil a déjà mis en exergue cette différence de situation, voir par exemple: CC, déc. nº 2011-19o QPC du 21 octobre 2011, M. Bruno L. et autres [Frais irrépétibles devant les juridictions pénales].

153. A. Botton, «Constitutionnalité de l’appel limité des parties civiles...», p. 652; voir également Y. Joseph-Ratineau, «Le droit d'appel limité des parties civiles et le Conseil constitutionnel», Gazette du palais, $\mathrm{n}^{\circ} 60,1^{\mathrm{er}}$ mars 2014, p. 869-872.

154. CC, déc. $\mathrm{n}^{\circ}$ 2004-492 DC du 2 mars 2004, Loi portant adaptation de la justice aux évolutions de la criminalité; CC, déc. $\mathrm{n}^{\circ}$ 2013-679 DC du 4 décembre 2013, Loi relative à la lutte contre la fraude fiscale et la grande délinquance économique et financière.

155. Il s'agit ici de délits d'atteinte aux biens, le Conseil opérant «une distinction entre les délits d'atteinte aux biens et les délits d'atteinte aux personnes "; voir J.-B. Perrier, "Le Conseil constitutionnel, l'escroquerie en bande organisée et la garde à vue: l'abstraction de l'inconstitutionnalité», L'actualité juridique. Droit pénal, $\mathrm{n}^{\circ} 12$, décembre 2014, p. 575.

156. CC, déc. no 2014-420/421 QPC du 9 octobre 2014, M. Maurice L. et autre [Prolongation exceptionnelle de la garde à vue pour des faits d'escroquerie en bande organisée], cons. 13

157. CC, déc. no 2014-428 QPC du 21 novembre 2014, M. Nadav B. [Report de l'intervention de l'avocat au cours de la garde à vue en matière de délinquance ou de criminalité organisées].

158. F. Desprez, "Conformité à la Constitution du report de l'intervention de l'avocat au cours de la garde à vue pour criminalité organisée», Lettre "Actualités Droits-Libertés» de La revue des droits de l'homme, 23 décembre 2014, en ligne: https://revdh.revues.org/1035.

159. CC, déc. nº 2014-693 DC du 25 mars 2014, cons. 23.

160. E. Bonis-Garçon, V. Peltier, «Chronique de droit pénal et procédure pénale», Les nouveaux cahiers du Conseil constitutionnel, n 44 , juin 2014 , p. 109.

161. D. Rousseau, Droit du contentieux constitutionnel, $10^{\mathrm{e}}$ éd., Paris, LGDJ-Lextenso, 2013, p. 481.

162. Voir F. Mélin-Soucramanien, «Le principe d'égalité dans la jurisprudence du Conseil constitutionnel. Quelles perspectives pour la question prioritaire de constitutionnalité ?», Les cahiers du Conseil constitutionnel, $\mathrm{n}^{\circ}$ 29, octobre 2010, en ligne: http://www.conseil-constitutionnel.fr/ conseil-constitutionnel/francais/nouveaux-cahiers-du-conseil/cahier-n-29/le-principe-d-egalite-dans-la-jurisprudence-du-conseil-constitutionnelquelles-perspectives-pour-la-question-prioritaire-de-constitutionnalite.52731.html.

163. F. Miclo, «Le principe d'égalité et la constitutionnalité des lois», L'actualité juridique. Droit administratif, 1982, p. 115. 
à une évolution constante. Cette diversité se vérifie encore cette année où le principe d'égalité s'applique par exemple en matière de droit local ${ }^{164}$, de droit du travail ${ }^{165}$, de droit électoral ${ }^{166}$, de droit pénal ${ }^{167}$, en matière économique ${ }^{168}$, civile $^{169}$, d'énergie et de droit des contrats ${ }^{170}$. Ce principe a été aussi évoqué dans le domaine politique ${ }^{171}$, des finances publiques ${ }^{172}$ et de la fiscalité.

Ainsi, l'égalité devant les charges publiques, principe reconnu depuis longtemps $s^{173}$ par le juge constitutionnel, a tenu une place importante dans sa jurisprudence cette année ${ }^{174}$. Aucune réelle évolution n'est intervenue en la matière concernant la position du Conseil ${ }^{175}$, un point intéressant sera néanmoins développé concernant le contrôle des dérogations qui peuvent être apportées à ce principe.

Le principe d'égalité sera abordé sous deux angles: le premier, général, consacré à l'utilisation fréquente par le Conseil de l'article 6 de la Déclaration de 1789 (1); le second, plus spécifique et relatif à la problématique de l'égalité hommes / femmes (2).

\section{Un traitement traditionnel}

et uniforme du principe d'égalité:

la référence à l'article 6 de la Déclaration des droits de l'homme et du citoyen de 1789

Le principe d'égalité ne s'oppose ni à ce que le législateur règle de façons différentes des situations différentes ni à ce qu'il déroge à l'égalité pour des raisons d'intérêt général, pourvu que, dans l'un et l'autre cas, la différence de traitement soit en rapport direct avec l'objet de la loi qui l'établit ${ }^{176}$.

Ce considérant de principe - rattaché à l'article 6 de la Déclaration de 1789 - est depuis longtemps utilisé par le juge constitutionnel dans le contrôle des justifications possibles des atteintes au principe d'égalité. Le Conseil a pu tirer de ce fondement deux «modes de contrôle»: celui fondé sur l'existence d'une différence de traitement correspondant à une différence de situation, et, celui d'une raison d'intérêt général justifiant la dérogation à l'égalité. Le critère commun à ces deux principes est bien sûr l'exigence d'un rapport direct avec l'objet de la loi, qui doit être présent dans tous les cas. Parfois, le juge constitutionnel analyse l'ensemble de ces motifs mais il arrive qu'il se focalise uniquement sur un de ces principes. Il va ainsi se concentrer soit sur une différence de situation soit sur l'existence d'une raison d'intérêt général. Tout dépend du cas d'espèce, ce qui témoigne d'autant plus de la liberté du Conseil en la matière.

Dans la plupart des QPC, le Conseil ne commence pas directement à examiner si la différence de traitement est en rapport direct avec la différence de situation ou avec l'objectif d'intérêt général poursuivi. En effet, le juge constitutionnel contrôle, avant tout, la rationalité et l'objectivité du critère sur lequel une différence de traitement est fondée. Cela implique qu'il soit défini par la loi et ne laisse pas aux autorités chargées de l'appliquer un pouvoir d'appréciation arbitraire ${ }^{177}$.

Le «motif» de la différence de situation a été largement utilisé cette année encore. Ainsi, le juge a décidé que la différence de traitement instaurée par la «loi garantissant l'avenir et la justice des systèmes de retraites » entre les salariés employés dans des conditions de droit privé bénéficiant d'un régime spécial de retraite et ceux n'en bénéficiant pas se fondait bien sur une différence de situation et partant n'était pas contraire au principe d'égalité ${ }^{178}$. Il s'est également fondé sur une différence de situation dans la décision nº 2014-

164. CC, déc. n²014-414 QPC du 26 septembre 2014.

165. CC, déc. $\mathrm{n}^{\circ}$ 2014-401 QPC du 13 juin 2014, Recours au contrat de travail à durée déterminée et exclusion du versement de l'indemnité de fin de contrats; CC, déc. n 2014-402 QPC du 13 juin 2014, Recours au contrat de travail à durée déterminée et exclusion du versement de l'indemnité de fin de contrats; CC, déc. no 2014-683 DC du 16 janvier 2014, Loi garantissant l'avenir et la justice du système des retraites.

166. CC, déc. $n^{\circ}$ 2014-405 QPC du 20 juin 2014, Commune de Salbris [Répartition des sièges de conseillers communautaires entre les communes membres d'une communauté de communes ou d'une communauté d'agglomérations]; CC, déc. n² 2014-407 QPC du 18 juillet 2014 ; CC, déc. n² $2014-687$ DC du 23 janvier 2014.

167. CC, déc. $\mathrm{n}^{\circ}$ 2014-427 QPC du 14 novembre 2014, Extradition des personnes ayant acquis la nationalité française.

168. CC, déc. $\mathrm{n}^{\mathrm{o}}$ 2014-422 QPC du 17 octobre 2014; CC, déc. nº 2014-698 du 6 août 2014. Dans cette décision le Conseil censure l'article $1^{\text {er }}$ du projet de «Pacte de responsabilité » qui prévoyait une baisse dégressive des cotisations salariales pour les salaires compris entre 1 et 1,3 SMIC.

169. CC, déc. $\mathrm{n}^{\circ}$ 2014-398 QPC du 2 juin 2014, Sommes non prises en considération pour le calcul de la prestation compensatoire.

170. CC, déc. n 2014-410 QPC du 18 juillet 2014, Société Roquette Frères.

171. CC, déc. $\mathrm{n}^{\circ}$ 2014-407 QPC du 18 juillet 2014 .

172. CC, déc. $\mathrm{n}^{\circ}$ 2014-698 DC du 6 août 2014; CC, déc. $\mathrm{n}^{\circ}$ 2014-706 DC du 18 décembre 2014

173. Ce principe, fondé sur l'article 13 de la Déclaration de 1789, a été consacré par le Conseil dans la décision de 1982. CC, déc. n $81-133$ DC du 30 décembre 1981, Loi de finances pour 1982, cons. 6.

174. CC, déc. nº 2014-419 QPC du 8 octobre 2014; CC, déc. n² 2014-431 QPC du 28 novembre 2014; CC, déc. n 2014-708 DC du 29 décembre 2014.

175. CC, déc. $\mathrm{n}^{\circ}$ 2014-698 DC du 6 août 2014. Ici le Conseil rappelle la différence entre les impositions de toute nature et les cotisations sociales; CC, déc. $\mathrm{n}^{\circ}$ 2014-707 DC du 29 décembre 2014: ici le Conseil réaffirme le régime particulier de l'impôt de solidarité sur la fortune (ISF) qui n'entre pas dans la catégorie des impositions de toute nature.

176. Formulation du principe d'égalité, en grande partie, issue de la décision CC, déc. $\mathrm{n}^{\circ}$ 87-232 DC du 7 janvier 1988, Loi relative à la mutualisation de la Caisse nationale du Crédit agricole, cons. 10. L'exigence de la nature «directe» du lien relève quant à elle de la décision CC, déc. $\mathrm{n}^{\circ} 97-388$ DC du 20 mars 1997, Loi créant les plans d'épargne retraite, cons. 27.

177. CC, déc. $\mathrm{n}^{\circ}$ 2014-402 QPC du 13 juin 2014. Dans cette décision le Conseil a pu reconnaître à deux reprises que les «usages» ou "traditions» constituent un critère objectif et rationnel sur lequel une différence de traitement peut se fonder. CC, déc. $n^{\circ} 2013-361$ du 28 janvier 2014, Consorts $P$. de B., cons. 10. Le Conseil débute son argumentation en affirmant que la distinction opérée par le Code civil, entre l'adoption plénière et l'adoption simple, constitue un critère objectif justifiant une différence de traitement entre celui qui est «adopté simple» - exclu de la perception de droits de mutation à titre gratuit - et ceux se situant dans d'autres liens de parenté.

178. CC, déc. nº 2014-683 DC du 16 janvier 2014. 
427 QPC du 14 novembre 2014 dans laquelle il a considéré que des dispositions autorisant l'extradition de personnes qui n'avaient pas la nationalité française au moment de la commission de l'infraction n'étaient pas contraires au principe d'égalité. Celles-ci se fondaient sur une différence de situation: différence entre ceux qui ont la nationalité française - exclus de ladite procédure d'extradition - et ceux qui n'en sont pas titulaires au moment de la commission de l'infraction. En plus d'une différence de situation justifiant cette disposition, celle-ci présente un rapport direct avec l'objet de la loi: faire obstacle à l'utilisation de la nationalité pour échapper à l'extradition. Le constat de l'existence ou non d'une différence de situation justifiant une différence de traitement a ainsi été assez présent dans la jurisprudence en $2014^{179}$. Traiter différemment des personnes placées dans des situations différentes n'est néanmoins nullement une obligation. C'est ce qu'affirme, là aussi depuis longtemps, le Conseil. Celui-ci l'a bien rappelé cette année ${ }^{180}$. Bien plus, le juge constitutionnel refuse parfois de contrôler si les situations couvertes par des règles et des régimes différents pourraient être comparables ${ }^{181}$, ce faisant il refuse d'opérer une vérification approfondie.

Le deuxième «motif» tiré du célèbre considérant est celui de l'existence d'un motif d'intérêt général. Dans ce cas, le Conseil, après avoir identifié l'objectif d'intérêt commun poursuivi par le législateur vérifie si la différence de traitement est bien en adéquation avec ce dernier ${ }^{182}$.

Parfois, le Conseil se focalise surtout sur la recherche d'un «lien direct» avec l'objectif poursuivi par le législateur. C'était le cas dans la décision n 2014-40o QPC du 6 juin 2014 relative aux procédures de contestations d'impositions et aux intérêts «moratoires » ou « de retard" que celles-ci peuvent entraîner. Selon le juge constitution- nel, en excluant de la possibilité d'une imputation sur les frais de garanties nécessaires à une action de contestation d'imposition des «intérêts de retards» causés par ladite action, et non des «intérêts moratoires", la disposition était dépourvue de lien direct avec l'objet de la $\operatorname{loi}^{183}$ : faire en sorte que les frais concernés ne puissent être maintenus à la charge du contribuable quand celui-ci s'acquitte de sa dette fiscale. Au regard de l'objectif poursuivi par le législateur, la différence de traitement n'était pas justifiée par un critère objectif et rationnel.

\section{Une innovation dans le cadre de la QPC: censure de l'infériorité des droits reconnus aux femmes}

L'innovation provient de la décision $n^{\circ}$ 2013-36o QPC du 9 janvier 2014 qui portait sur la perte de la nationalité et sur l'égalité entre les sexes. En l'espèce, la requérante avait formé devant la Cour de cassation un pourvoi assorti d'une QPC estimant que les articles $87^{184}$ et $9^{185}$ de l'ordonnance $\mathrm{n}^{\circ}$ 45-2441 du 19 octobre 1945 - dont la rédaction actuelle provient de la loi du 9 avril 1954 - étaient contraires à la Constitution. La requérante contestait le fait que l'acquisition volontaire (hors mariage) d'une nationalité étrangère entraîne une perte automatique de la nationalité française pour les femmes et pas pour les hommes. Ainsi, en vertu de l'article 9, ils ne perdaient la nationalité qu'avec autorisation du gouvernement. Le Conseil donne raison à la requérante en censurant les mots «sexe masculin» des premier et troisième alinéas de l'article 9 de ladite ordonnance. La QPC commentée innove à double titre.

D'une part, c'est la première fois ${ }^{186}$ que le Conseil est saisi d'un grief dénonçant l'infériorité de droits reconnus aux femmes. En effet, dans le domaine de l'égalité

179. CC, déc. n² 2014-706 DC du 18 décembre 2014; CC, déc. n²013-365 QPC du 6 février 2014, Époux M.

180. CC, déc. $\mathrm{n}^{\circ}$ 2014-422 QPC du 17 octobre 2014. Dans cette décision était contestée la mise en place de techniques électroniques de réservations préalables pour les véhicules de tourisme. Un syndicat représentant les intérêts des «taxis» considérait que de telles mesures les mettaient, de manière injustifiée, en concurrence avec les véhicules de tourisme. Les premiers étaient, en effet, soumis à une réglementation plus exigeante relative à l'accès à la profession. Ils réclamaient ainsi qu'une différence de traitement soit opérée entre eux et les véhicules de tourisme, ce que refusera le Conseil: des situations différentes n'entraînent pas obligation d'une différenciation de traitement (CC, déc. nº $2014-696$ DC du 7 août 2014, cons. 17).

181. Cela ressort d'une jurisprudence constante du Conseil qui se refuse d'opérer tout contrôle approfondi de l'égalité entre des règles de droit privé et des règles de droit public sous le prétexte que les situations que ces différentes règles couvrent pourraient être comparables. Cela est vrai pour la comparaison tant des régimes de travail que pour ceux de Sécurité sociale: CC, déc. n²014-686 DC du 23 janvier 2014, cons. 10. Cela se vérifie aussi dans le domaine fiscal: CC, déc. $\mathrm{n}^{\circ} 2014-425$ QPC du 14 novembre 2014.

182. CC, déc. $\mathrm{n}^{\circ}$ 2014-410 du 18 juillet 2014, dans laquelle était en cause une disposition de l'article L. 314-1 du Code de l'énergie qui réservait le bénéfice de l'obligation d'achat aux installations de cogénération d'une puissance qui n'excède pas $12 \mathrm{MW}$. Le juge constitutionnel a considéré ici que le motif d'intérêt général n'était pas en adéquation avec la différence de traitement. Pour décider cela, le Conseil a d'abord examiné l'objet de la loi et a considéré que l'exclusion des installations de plus de $12 \mathrm{MW}$ n'était pas justifiée car elles peuvent aussi concourir aux objectifs visés par la loi: ceux de l'efficacité énergétique et de la sécurité des approvisionnements que permet la cogénération; CC, déc. n $2014-407$ QPC du 18 juillet 2014.

183. CC, déc. nº 2014-40o du 6 août 2014, Société Orange SA, cons. 8. On retrouve ce fondement, CC, déc. n 2014-398 QPC du 2 juin 2014, cons. 9. Le Conseil ici considère que l'exclusion dans le calcul de la prestation compensatoire, sur le fondement de l'article 272 du Code civil, des sommes versées à l'un des époux au titre de la réparation d'un accident du travail ou au titre de la compensation d'un handicap «institue entre les époux une différence de traitement qui n'est pas en rapport avec l'objet de la prestation compensatoire» qui est de compenser la disparité dans les conditions de vie respectives entraînée par la rupture du mariage.

184. Article 87: «Perd la nationalité française le Français majeur qui acquiert volontairement une nationalité étrangère».

185. Article 9: «Jusqu’à une date qui sera fixée par décret, l'acquisition d'une nationalité étrangère par un Français du sexe masculin ne lui fait perdre la nationalité française qu'avec l'autorisation du gouvernement français. Cette autorisation est de droit lorsque le demandeur a acquis une nationalité étrangère après l'âge de cinquante ans».

186. Il faut néanmoins nuancer ce propos. Le grief posé n'était, dans son objet, cependant pas totalement nouveau dans la mesure où une même QPC avait été transmise en avril 2012 à la Cour de cassation qui cependant ne l'avait pas renvoyée au Conseil au motif que la disposition contestée n'étai pas applicable au litige. La nouveauté réside ici donc dans le fait que c'est le premier grief de cet objet qui arrive jusqu'au Conseil constitutionnel. En effet, jusqu'ici, celui-ci avait été saisi surtout de griefs qui concernaient la parité hommes / femmes. 
hommes / femmes, le Conseil avait surtout eu à connaître de dispositions qui visaient à améliorer la situation de la femme par rapport à une situation de droit d'inégalité originaire $^{187}$. Ici ce n'est pas une mesure de «discrimination positive ${ }^{188}$ qui était en jeu mais une mesure de « discrimination", qui prévoyait un statut différent entre l'homme et la femme quant au maintien de la nationalité française. Le Conseil poursuit donc l'évolution de sa jurisprudence en matière d'égalité hommes / femmes en abordant une autre face de ce principe, celle de «l'inégalité» entre un homme et une femme. Le Conseil accueille le grief en censurant la loi en question. Ce faisant, celui-ci explore une dimension nouvelle, bien qu'évidente ${ }^{189}$, de sa mission de protection de l'égalité hommes / femmes.

D'autre part, il semble intéressant d'identifier le fondement sur lequel le Conseil raisonne. Il ne s'appuie pas seulement sur l'article 6 de la Déclaration des droits de l'homme et du citoyen du 14 août 1789 mais aussi sur le troisième alinéa du préambule de 1946: «La loi garantit à la femme dans tous les domaines, des droits égaux à ceux de l'homme». Cela est marquant dans la mesure où le Conseil n'avait encore jamais rattaché le principe d'égalité à cette disposition. Il n'est pas impossible d'en déduire un renouvellement, voire un renforcement de son contrôle, dans le domaine de l'égalité hommes / femmes. Lorsque le Conseil examine la constitutionnalité d'une loi et que le principe d'égalité hommes / femmes est invoqué, il ne se fonderait plus seulement sur l'article 6 de la Déclaration des droits mais aussi sur le troisième alinéa du préambule de 1946. La différence de traitement doit donc non seulement répondre aux exigences posées dans le célèbre considérant de principe, fondé sur l'article 6 de la Déclaration, mais doit aussi être justifiée au regard des exigences portées par le troisième alinéa du préambule de 1946. C’est sur le «détail» de la combinaison opérée en l'espèce que subsiste une certaine part d'ombre. En effet, le Conseil utilise cette référence mais en la citant simplement et sans préciser la place que cette disposition a tenue dans son contrôle. Il censure la loi au motif que la disposition institue une «différence de traitement sans rapport avec l'objectif poursuivi et qui ne peut être regardée comme justifiée ${ }^{190}$. On voit donc que son raisonnement dans le fond repose en grande partie sur les principes qu'il fait découler depuis longtemps de l'article 6 de la Déclaration de 1789. Il est difficile aujourd'hui de mesurer la répercussion de ce «contrôle intensifié» sur la jurisprudence future.

187. Dans le domaine de l'égalité hommes / femmes, le Conseil avait, jusqu'ici, eu à connaître uniquement de dispositions qui instauraient des mesures de discrimination positive en faveur des femmes.

188. En la matière, le juge constitutionnel n'accepte que depuis récemment, et sous certaines conditions, de reconnaître la constitutionnalité de mesures de discrimination positive. Les révisions constitutionnelles du 8 juillet 1999 et du 23 juillet 2008 , modifiant sur ce point l'article $1^{\text {er }}$ de la Constitution, en ont d'ailleurs été de véritables moteurs. Le Conseil a ainsi validé certaines mesures de discrimination positive. Voir par exemple, CC, déc. $\mathrm{n}^{\circ}{ }_{2003-483}$ DC du 14 août 2003, Loi portant réforme des retraites; CC, déc. n $2012-658$ DC du 13 décembre 2012 , Loi organique relative à la programmation et à la gouvernance des finances publiques. Le Conseil, ici, a jugé conformes à la Constitution des dispositions qui instauraient une représentation paritaire des hommes et des femmes au sein du Haut Conseil des finances publiques.

189. Dans la mesure où toute distinction fondée sur le sexe est considérée à l'origine comme étant absolument prohibée par la Constitution. Ce critère fait traditionnellement partie avec les critères de l'origine, de la race, de la religion et des croyances des éléments qui ne peuvent servir de fondement à une différence de traitement. Toute discrimination fondée sur ces critères est strictement interdite, cela résulte de l'article $1^{\text {er }}$ de la Constitution du 4 octobre 1958 et de l'alinéa 3 du préambule de la Constitution du 27 octobre 1946.

190. CC, déc. $n^{\circ}$ 2014-36o du 9 janvier 2014, $M^{m e}$ Jalika K., cons. 8. 